\title{
REFLECTION OF STATIONARY SETS AND THE TREE PROPERTY AT THE SUCCESSOR OF A SINGULAR CARDINAL
}

\author{
LAURA FONTANELLA AND MENACHEM MAGIDOR
}

\begin{abstract}
We show that from infinitely many supercompact cardinals one can force a model of ZFC where both the tree property and the stationary reflection hold at $\aleph_{\omega^{2}+1}$.
\end{abstract}

$\S 1$. Introduction. One of the most fruitful research areas in set theory concerns the investigation of models of set theory where properties of large cardinals hold at small cardinals. The results presented in this paper focus on two properties of weakly compact cardinals that, under large cardinal assumptions, can be forced at small cardinals, the tree property and the reflection of stationary sets. To get a model of the tree property at the double successor of a regular cardinal it is enough to assume the consistency of a weakly compact cardinal and force with a classical poset due to Mitchell (see [6]). Forcing the tree property at the successor of a singular cardinal is harder and requires much stronger assumptions. The first model of the tree property at the successor of a singular cardinal, was defined by Magidor and Shelah in [4] who proved from large cardinals the consistency of the tree property at $\aleph_{\omega+1}$. The hypotheses used in such result have the consistency strength of a large cardinal between a huge cardinal and a 2-huge cardinal; this was later improved by Sinapova [10] who was able to force the tree property at $\aleph_{\omega+1}$ from weaker large cardinal assumptions, namely assuming the consistency of infinitely many supercompact cardinals. Another paper by Neeman [7] shows that, assuming the consistency of infinitely many supercompact cardinals, one can force a model where the tree property holds simultaneously at $\aleph_{\omega+1}$ and at every $\aleph_{n}$ with $n \geq 2$. All these constructions can be adapted to force the tree property at the successor of any singular cardinal $\kappa$ of countable cofinality, however in all these models the reflection of stationary sets fails at $\kappa^{+}$. In the case of $\aleph_{\omega+1}$, for instance, these forcing constructions all add a bad scale. So it is natural to ask whether the tree property and the reflection of stationary sets are incompatible at the successor of a singular cardinal. In this paper we answer this question for a particular cardinal, namely $\aleph_{\omega^{2}+1}$. We show that, assuming the consistency of infinitely many supercompact cardinals, one can force a model where both the tree property and the stationary

Received August 25, 2015.

2010 Mathematics Subject Classification. $03 \mathrm{E} 05$.

Key words and phrases. reflection of stationary sets, tree property, large cardinals.

(C) 2017, Association for Symbolic Logic 0022-4812/17/8201-0016 DOI: $10.1017 /$ jsl.2016.13 
set reflection hold at $\aleph_{\omega^{2}+1}$. Whether the same can be proven for $\aleph_{\omega+1}$ remains an open problem.

We will use a forcing construction due to Magidor and Shelah [5] that was introduced to define a model where $\aleph_{\omega^{2}+1}$ satisfies a strong reflection principle, denoted $\Delta_{\aleph_{\omega^{2}}, \aleph_{\omega^{2}+1}}$. The principle is defined as follows.

Definition 1.1. Given two cardinals $\kappa<\lambda, \Delta_{\kappa, \lambda}$ is the statement that for every cardinal $\mu<\kappa$, for every stationary set $S \subseteq E_{<\kappa}^{\lambda}:=\{\alpha<\lambda$; $\operatorname{cof}(\alpha)<\kappa\}$ and for every algebra $A$ on $\lambda$ with $\mu$ operations, there exists a subalgebra $A^{\prime}$ of order type a regular cardinal $\eta<\kappa$ such that $S \cap A^{\prime}$ is stationary in $\sup \left(A^{\prime}\right)$.

Let us denote by $\Delta_{<\lambda, \lambda}$ the principle $\forall \kappa<\lambda \Delta_{\kappa, \lambda}$. Under this principle it is possible to prove several 'compactness' results, namely theorems where, given a structure of size $\lambda$, properties of substructures of size $\leq \kappa$ imply a global property for the whole structure. For instance, assuming $\Delta_{<\lambda, \lambda}$ one can prove that every almost free Abelian group of size $\lambda$ is free (where 'almost free' means that every subgroup of smaller size is free). Magidor and Shelah proved in [5] that assuming the consistency of infinitely many supercompact cardinals, there exists a model of ZFC $+\mathrm{GCH}$ where $\Delta_{\aleph_{\omega^{2}}, \aleph_{\omega^{2}+1}}$ holds. The results presented in [5] combined with other previous results (see [1], [8], [9]) showed also that $\aleph_{\omega^{2}+1}$ is the smallest regular cardinal $\lambda$ that can consistently satisfy $\Delta_{<\lambda, \lambda}$. In this paper we prove that in the Magidor-Shelah's model, $\aleph_{\omega^{2}+1}$ satisfies even the tree property.

THEOREM 1.2. Assuming the consistency of infinitely many supercompact cardinals, there exists a model of ZFC where both $\Delta_{\aleph_{\omega^{2}}, \aleph_{\omega^{2}+1}}$ and the tree property at $\aleph_{\omega^{2}+1}$ hold.

The principle $\Delta_{\kappa, \lambda}$ expresses a strong form of reflection, in particular $\Delta_{<\lambda, \lambda}$ implies the reflection of stationary subsets of $\lambda$. It follows from Theorem 1.2 that, assuming the consistency of infinitely many supercompact cardinals, $\aleph_{\omega^{2}+1}$ can consistently satisfy both the tree property and $\Delta_{\aleph_{\omega^{2}}, \aleph_{\omega^{2}+1}}$, hence the stationary set reflection.

In the second part of this paper we show that $\Delta_{\aleph_{\omega^{2}}, \aleph_{\omega^{2}+1}}$ does not imply the tree property at $\aleph_{\omega^{2}+1}$. More precisely, we prove the following theorem.

THEOREM 1.3. Assuming the consistency of infinitely many supercompact cardinals, we can force a model of ZFC where $\Delta_{\aleph_{\omega^{2}}, \aleph_{\omega^{2}+1}}$ holds while the tree property fails at $\aleph_{\omega^{2}+1}$.

The paper is organized as follows. In Section 2 we recall some classical results that will be used repeatedly in the proofs of Theorems 1.2 and 1.3. In Sections 3 and 4 we present Magidor-Shelah's forcing construction for building a model of $\Delta_{\aleph_{\omega^{2}}, \aleph_{\omega^{2}+1}}$. In Section 5 we prove that in the Magidor-Shelah's model the tree property holds at $\aleph_{\omega^{2}+1}$, so we prove Theorem 1.2. Finally, Section 6 is devoted to the proof of Theorem 1.3.

§2. Preliminaries. In this section we list some classical results about forcing that preserve Aronszajn trees. We recall that a $\kappa$-Aronszajn tree is a $\kappa$-tree with no cofinal branches. To simplify the notation, we will always assume that our $\kappa^{+}$-trees are subsets of $\kappa^{+} \times \kappa$, and that for every $\alpha<\kappa^{+}$the $\alpha$-th level of the tree $\operatorname{Lev}_{\alpha}(T)$ is a subset of $\{\alpha\} \times \kappa$.

A simple argument shows that if $T$ is a $\kappa$-Aronszajn tree and $\mathbb{P}$ is a $\kappa$-Knaster forcing notion, then $T$ remains Aronszajn after forcing with $\mathbb{P}$ : otherwise, if $\dot{b}$ is a 
name for a cofinal branch on $T$, then we can pick for every $\alpha<\kappa$ a condition $p_{\alpha}$ deciding the value of $\dot{b} \cap \alpha$. By the Knaster property there exists a cofinal subset $I \subseteq \kappa$ such that the conditions in the sequence $\left\langle p_{\alpha} ; \alpha \in I\right\rangle$ are pairwise compatible. Say that $p_{\alpha} \Vdash \dot{b} \cap \alpha=t_{\alpha}$ for each $\alpha$, then $\left\{t_{\alpha} ; \alpha \in I\right\}$ is a cofinal branch for $T$, contradicting $T$ is $\kappa$-Aronszajn. The following lemma by Unger show that for a forcing $\mathbb{P}$ to preserve $\kappa$-Aronszajn trees it is enough that $\mathbb{P} \times \mathbb{P}$ has the $\kappa$-chain condition.

Lemma 2.1 (Unger [11]). Let $\mathbb{P}$ be a forcing notion such that $\mathbb{P} \times \mathbb{P}$ is $\kappa$-c.c. Then $\mathbb{P}$ has the $\kappa$-approximation property, i.e., given a set of ordinals $A$ in a $\mathbb{P}$-generic extension $V[G]$, if $A \cap x \in V$ for every $x \in V$ of size $<\kappa$, then $A \in V$. In particular, if $T$ is a $\kappa$-tree and $\mathbb{P} \times \mathbb{P}$ is $\kappa$-c.c., then forcing with $\mathbb{P}$ does not add cofinal branches to $T$.

Proof. Suppose for a contradiction that for some ordinal $\tau$ there exists a $\mathbb{P}$-name $\dot{A}$ such that

$$
\Vdash_{\mathbb{P}} \dot{A} \subseteq \tau, \forall x \in[\tau]^{<\kappa}(\dot{A} \cap x \in V) \text { and } \dot{A} \notin V .
$$

We inductively define conditions $\left\langle p_{i}^{0}, p_{i}^{1}\right\rangle_{i<\kappa}$ in $\mathbb{P} \times \mathbb{P}$, sets $\left\langle d_{i}^{0}, d_{i}^{1}\right\rangle_{i<\kappa}$ in $[\tau]^{<\kappa}$ and a $\subseteq$-strictly increasing sequence $\left\langle x_{i}\right\rangle_{i<\kappa}$ in $[\tau]^{<\kappa}$ such that

(1) for $\varepsilon \in\{0,1\}, p_{i}^{\varepsilon} \Vdash \dot{A} \cap x_{i}=d_{i}^{\varepsilon}$;

(2) $d_{i}^{0} \neq d_{i}^{1}$ and $d_{i}^{0} \cap\left(\bigcup_{j<i} x_{j}\right)=d_{i}^{1} \cap\left(\bigcup_{j<i} x_{j}\right)$.

Suppose we have constructed $\left\langle p_{i}^{0}, p_{i}^{1}\right\rangle_{i<j},\left\langle d_{i}^{0}, d_{i}^{1}\right\rangle_{i<j}$ and $\left\langle x_{i}\right\rangle_{i<j}$ successfully. Let $x:=\bigcup_{i<j} x_{i}$ and let $p$ be any condition in $\mathbb{P}$ deciding the value of $\dot{A} \cap x$ to be $d \in[\tau]^{<\kappa}$. As $\dot{A}$ does not belong to $V$, we can find $p_{j}^{0}, p_{j}^{1} \leq p$, distinct $d_{j}^{0}, d_{j}^{1}$ and $x_{j} \supset x$ such that $p_{j}^{\varepsilon} \Vdash \dot{A} \cap x_{j}=d_{j}^{\varepsilon}$ for $\varepsilon \in\{0,1\}$. Then $p_{j}^{\varepsilon} \Vdash d=\dot{A} \cap x=d_{j}^{\varepsilon} \cap x$ hence $d_{j}^{0} \cap x=d=d_{j}^{1} \cap x$.

Now we claim that $\left\langle p_{i}^{0}, p_{i}^{1}\right\rangle_{i<\kappa}$ is an antichain, contradicting the $\kappa$-chain condition at $\mathbb{P} \times \mathbb{P}$. Suppose that for some $i<j$, the conditions $\left(p_{i}^{0}, p_{i}^{1}\right)$ and $\left(p_{j}^{0}, p_{j}^{1}\right)$ are compatible. Then $d_{j}^{0} \cap x_{i}=d_{i}^{0}$ and $d_{j}^{1} \cap x_{i}=d_{i}^{1}$. By construction $d_{j}^{0} \cap\left(\bigcup_{l<j} x_{l}\right)=$ $d_{j}^{1} \cap\left(\bigcup_{l<j} x_{l}\right)$, in particular $d_{j}^{0} \cap x_{i}=d_{j}^{1} \cap x_{i}$, contradicting $d_{i}^{0} \neq d_{i}^{1}$.

The following results by Magidor and Shelah shows that forcings with $\sigma$-closure preserve Aronszajn trees over successors of singular cardinals of countable cofinality.

Lemma 2.2 (Magidor and Shelah [4, Theorem 5.2]). Let $\lambda$ be a singular cardinal of countable cofinality and let $T$ be $a \lambda^{+}$-tree. Assume that $\mathbb{P}$ is a $\sigma$-closed poset, then forcing with $\mathbb{P}$ does not add cofinal branches to $T$.

Proof. Let $\dot{b}$ be a $\mathbb{P}$-name for a cofinal branch of $T$, and suppose for a contradiction that such a branch is not in $V$. We can inductively define for every $n<\omega$, conditions $\left\langle p_{s} ; s \in{ }^{n} \lambda\right\rangle$ and ordinals $\left\langle\alpha_{s} ; s \in{ }^{n} \lambda\right\rangle$ in $\lambda^{+}$such that

(1) $s \sqsubseteq t$ implies $p_{t} \leq p_{s}$,

(2) $p_{s \frown 0}$ and $p_{s \frown 1}$ force contradictory information about $\dot{b} \cap \operatorname{Lev}_{\alpha_{s}}(T)$.

Then, we let $\alpha$ be the limit of all $\alpha_{s}$ and, for each $f \in{ }^{\omega} \lambda$, we let $p_{f}$ be a lower bound for the sequence $\left\langle p_{f \nmid n} ; n<\omega\right\rangle$ that decides the value of $\dot{b} \cap \operatorname{Lev}_{\alpha}(T)$ as $x_{f}$. By construction the $x_{f}$ 's are pairwise distinct. This implies that $\operatorname{Lev}_{\alpha}(T)$ has size $\lambda^{\omega}$, a contradiction. 
When we work with a $\kappa^{+}$-tree $\dot{T}$ in a generic extension by some forcing notion $\mathbb{P}$, it is often useful to consider the following relations $<_{p}$ on $\kappa^{+} \times \kappa$ where $p \in \mathbb{P}$ : for $(\alpha, \zeta),(\beta, \eta) \in \kappa^{+} \times \kappa$, we let $(\alpha, \zeta)<_{p}(\beta, \eta)$ when $p \Vdash(\alpha, \zeta)<_{T}(\beta, \eta)$. This lead us to the notion of system introduced by Magidor and Shelah in [5].

Definition 2.3 (Magidor and Shelah [5]). Let $D$ be a set of ordinals and $\tau$ a cardinal. A system over $D \times \tau$ is a collection of transitive, reflexive relations $\left\{R_{i}\right\}_{i \in I}$ on $D \times \tau$ such that:

(1) if $(\alpha, \zeta) R_{i}(\beta, \eta)$ and $(\alpha, \zeta) \neq(\beta, \eta)$, then $\alpha<\beta$;

(2) if $\left(\alpha_{0}, \zeta_{0}\right)$ and $\left(\alpha_{1}, \zeta_{1}\right)$ are both below $(\beta, \eta)$ in $R_{i}$, then $\left(\alpha_{0}, \zeta_{0}\right)$ and $\left(\alpha_{1}, \zeta_{1}\right)$ are comparable in $R_{i}$ (by condition (1) this implies that $\left(\alpha_{0}, \zeta_{0}\right) R_{i}\left(\alpha_{1}, \zeta_{1}\right)$ if $\alpha_{0}<\alpha_{1},\left(\alpha_{1}, \zeta_{1}\right) R_{i}\left(\alpha_{0}, \eta_{0}\right)$ if $\alpha_{1}<\alpha_{0}$, and $\zeta_{0}=\zeta_{1}$ if $\left.\alpha_{0}=\alpha_{1}\right)$;

(3) for every $\alpha<\beta$ both in $D$, there is $i \in I$ and $\zeta, \eta \in \tau$ such that $(\alpha, \zeta) R_{i}(\beta, \eta)$.

If $\mathscr{R}:=\left\{R_{i}\right\}_{i \in I}$ is a system over $D \times \tau$, then every element of $D \times \tau$ is a node of $\mathscr{R}$. For every $\alpha \in D$, the $\alpha$-th level of $\mathscr{R}$, denoted $\operatorname{Lev}_{\alpha}(\mathscr{R})$, is the set $\{\alpha\} \times \kappa$.

Definition 2.4 (Sinapova [10]). Let $\left\{R_{i}\right\}_{i \in I}$ be a system on $D \times \tau$. A branch through some $R_{i}$ is a partial function from $b: D \rightarrow \tau$ such that for any $\beta \in$ $\operatorname{dom}(b)$ and any $\alpha<\beta$ in $D, \alpha \in \operatorname{dom}(b)$ if and only if there exists $\zeta$ such that $(\alpha, \zeta) R_{i}(\beta, b(\beta))$ and $b(\alpha)$ is equal to the unique $\zeta$ witnessing this $(\zeta$ is unique by condition (2) of the definition of system). We say that $b$ is a cofinal if $\operatorname{dom}(b)$ is cofinal in $D$.

Sinapova proved a useful preserving theorem for systems.

TheORem 2.5 (Sinapova [10]). Suppose that $v$ is a singular cardinal of countable cofinality and $\mathscr{R}=\left\{R_{i}\right\}_{i \in I}$ is a system on $D \times \tau$ with $D$ cofinal in $v^{+}$ and $\max (|I|, \tau)<v$. Suppose that $\mathbb{P}$ is a $\chi$-closed forcing notion with $\chi>\max (\tau,|I|)^{+}$ and $G$ is a generic filter for $\mathbb{P}$ over $V$. Suppose that in $V[G]$ there are (not necessarily all cofinal) branches $\left\langle b_{i, \delta} ; i \in I, \delta<\tau\right\rangle$ such that

(1) every $b_{i, \delta}$ is an $R_{i}$-branch, and for some $(i, \delta), b_{i, \delta}$ is cofinal;

(2) for all $\alpha \in D$, there is $(i, \delta)$ such that $\operatorname{Lev}_{\alpha}(\mathscr{R}) \cap b_{i, \delta}$ is non empty.

Then $\mathscr{R}$ has a cofinal branch in $V$.

§3. The Main forcing. In this section we present Magidor-Shelah's forcing construction for building models of $\Delta_{\aleph_{\omega^{2}}, \aleph_{\omega^{2}+1}}$. We assume that $\left\langle\kappa_{n}\right\rangle_{n<\omega}$ is an increasing sequence of supercompact cardinals which are indestructible by directed closed forcings (i.e., if $\mathbb{P}$ is a $\kappa_{n}$-directed closed forcing notion, then $\kappa_{n}$ is still supercompact in $V^{\mathbb{P}}$, see Laver [3]). We let $\lambda:=\lim _{n<\omega} \kappa_{n}$ and we assume that, for every $n<\omega$, we have $2^{\kappa_{n}}=\kappa_{n}^{+}$. For every $n<\omega$, we let

$$
\mathbb{S}_{n}:=\prod_{m \geq n} \operatorname{Coll}\left(\kappa_{m}^{+2},<\kappa_{m+1}\right) .
$$

Since every $\mathbb{S}_{n}$ is $\kappa_{n}$-directed closed, $\kappa_{n}$ remains supercompact in $V^{\mathbb{S}_{n}}$ so we can fix, for every $n<\omega$, an $\mathbb{S}_{n}$-term $\dot{F}_{n}$ for a normal ultrafilter on $\mathcal{P}_{\kappa_{n}}\left(\lambda^{+}\right)$in $V^{\mathbb{S}_{n}}$. $\dot{F}_{n}$ has a natural projection to a normal ultrafilter $U_{n}$ on $\kappa_{n}$. The poset $\mathbb{S}_{n}$ is actually $\kappa_{n}^{+2}$-closed and we assumed $2^{\kappa_{n}}=\kappa_{n}^{+}$, therefore forcing with $\mathbb{S}_{n}$ does not introduce new sets which are hereditarily of size $\leq \kappa_{n}^{+}$, hence we have $U_{n} \in V$. 
We let $\pi_{n}: V \rightarrow N_{n}$ be the elementary embedding corresponding to $U_{n}$. Consider $\operatorname{Coll}^{N_{n}}\left(\kappa_{n}^{+\omega+2},<\pi_{n}\left(\kappa_{n}\right)\right)$, this forcing has the $\pi_{n}\left(\kappa_{n}\right)$-chain condition in $N_{n}$ and $\pi\left(\kappa_{n}\right)$ is inaccessible. Therefore, there are $\pi_{n}\left(\kappa_{n}\right)$ many dense subsets of this forcing which are in $N_{n}$. On the other hand $\left|\pi_{n}\left(\kappa_{n}\right)\right|=\kappa_{n}^{+}$and the forcing is $\kappa_{n}^{+}$-closed in $N_{n}$. Therefore, one can inductively define in $V$ a generic filter $K_{n}$ for $\operatorname{Coll}^{N_{n}}\left(\kappa_{n}^{+\omega+2}\right.$, $<\pi_{n}\left(\kappa_{n}\right)$ ) over $N_{n}$ by meeting each dense set in $N_{n}$. We define the main forcing $\mathbb{P}$.

Definition 3.1. Conditions of $\mathbb{P}$ are sequences of the form

$$
p=\left\langle\alpha_{0}, g_{0}, f_{0}, \ldots, \alpha_{n-1}, g_{n-1}, f_{n-1}, A_{n}, g_{n}, F_{n}, \ldots\right\rangle
$$

such that:

(1) every $\alpha_{i}$ is an inaccessible cardinal between $\kappa_{i-1}$ and $\kappa_{i}\left(\right.$ with $\left.\kappa_{-1}:=\omega\right)$;

(2) $g_{0} \in \operatorname{Coll}\left(\omega, \alpha_{0}^{+\omega}\right)$ and for $0<i<n, g_{i} \in \operatorname{Coll}\left(\kappa_{i-1}^{++},<\alpha_{i}\right)$;

(3) $f_{i} \in \operatorname{Coll}\left(\alpha_{i}^{+\omega+2},<\kappa_{i}\right)$;

(4) $A_{j} \in U_{j}$ and every element of $A_{j}$ is an inaccessible cardinals;

(5) for $j \geq n, g_{j} \in \operatorname{Coll}\left(\kappa_{j-1}^{++},<\alpha\right)$ for the least $\alpha$ in $A_{j}$ (hence for every $\left.\alpha \in A_{j}\right)$;

(6) $F_{j}$ is a function with domain $A_{j}$ such that $F_{j}(\alpha) \in \operatorname{Coll}\left(\alpha^{+\omega+2},<\kappa_{j}\right)$ for every $\alpha \in A_{j}$, and such that $\left[F_{j}\right]_{U_{j}} \in K_{j}\left(\left[F_{j}\right]_{U_{j}}\right.$ is the equivalence class of $F_{j}$ as a member of the ultrapower $\left.\operatorname{Ult}\left(V, U_{j}\right)\right)$.

Given two conditions

$$
\begin{gathered}
p=\left\langle\alpha_{0}^{p}, g_{0}^{p}, f_{0}^{p}, \ldots, \alpha_{n-1}^{p}, g_{n-1}^{p}, f_{n-1}^{p}, A_{n}^{p}, g_{n}^{p}, F_{n}^{p}, \ldots\right\rangle \\
q=\left\langle\alpha_{0}^{q}, g_{0}^{q}, f_{0}^{q}, \ldots, \alpha_{m-1}^{q}, g_{m-1}^{q}, f_{m-1}^{q}, A_{m}^{q}, g_{m}^{q}, F_{m}^{q}, \ldots\right\rangle
\end{gathered}
$$

we say that $p \leq q$ if and only if, the following hold

(1) $m \leq n$ and for $i<m, \alpha_{i}^{p}=\alpha_{i}^{q}$ and $f_{i}^{p} \leq f_{i}^{q}$;

(2) for every $i<\omega, g_{i}^{p} \leq g_{i}^{q}$;

(3) for $m \leq j<n, \alpha_{j}^{p} \in A_{i}^{q}$ and $f_{i}^{p} \leq F_{i}^{q}\left(\alpha_{j}^{p}\right)$;

(4) for $j \geq n, A_{j}^{p} \subseteq A_{j}^{q}$ and $F_{j}^{p}(\alpha) \leq F_{j}^{q}(\alpha)$ for all $\alpha \in A_{j}^{p}$.

The only difference with the forcing defined in [5] is in the definition of the $g_{0}$ coordinates. Magidor and Shelah showed that forcing with $\mathbb{P}$ determines a model of $\Delta_{\aleph_{\omega^{2}}, \aleph_{\omega^{2}+1}}$, so in order to prove Theorem 1.2 we just need to show that there exists a generic extension by $\mathbb{P}$ where the tree property holds at $\aleph_{\omega^{2}+1}$.

Given a condition

$$
p=\left\langle\alpha_{0}, g_{0}, f_{0}, \ldots, \alpha_{n-1}, g_{n-1}, f_{n-1}, A_{n}, g_{n}, F_{n}, \ldots\right\rangle
$$

we say that

(1) $n$ is the length of $p$, and we denote it $\lg (p)$;

(2) the subsequence $\left\langle\alpha_{0}, g_{0}, f_{0}, \ldots, \alpha_{n-1}, g_{n-1}, f_{n-1}\right\rangle$ is called the lower part of $p$ or the stem of $p$, denoted stem $(p)$;

(3) $\left\langle\alpha_{0}, \ldots, \alpha_{n-1}\right\rangle$ is the $\alpha$-part of $p$;

(4) $\left\langle g_{0}, \ldots, g_{n-1}\right\rangle$ is the $g$-part of $p$;

(5) $\left\langle f_{0}, \ldots, f_{n-1}\right\rangle$ is the $f$-part of $p$;

(6) $\left\langle A_{j}: j \geq n\right\rangle$ is the $A$-part of $p$;

(7) $\left\langle g_{j}: j \geq n\right\rangle$ is the $S$-part of $p$ and for $k \geq n,\left\langle g_{j}: j \geq k\right\rangle$ is the $S_{k}$-part of $p$;

(8) $\left\langle F_{j}: j \geq n\right\rangle$ is the $F$-part of $p$; 
Given two conditions $p$ and $q$, we write $p \leq_{k} q$ when $p \leq q, \lg (p)=\lg (q)$, $p \nmid k=q\left\lceil k\right.$ and $g_{k}^{p}=g_{k}^{q}$.

$\S 4$. Basic properties of $\mathbb{P}$. We list some basic properties of $\mathbb{P}$.

Proposition 4.1 (Magidor Shelah [5, Lemmas 2 and 3]). The following hold for $\mathbb{P}$ :

(1) every $\leq_{n}$-decreasing sequence $\left\langle p_{\zeta}: \zeta<\eta\right\rangle$ of less than $\kappa_{n}$-many conditions each of length $n$ has a lower bound;

(2) every $\leq_{k}$-decreasing sequence $\left\langle p_{\zeta}: \zeta<\eta\right\rangle$ of at most $\alpha_{k}^{p_{0}}$-many conditions each of length $n>k$ has a lower bound;

(3) $\mathbb{P}$ satisfies the Prikry property in the following version: given a formula $\varphi$ and a condition $p \in \mathbb{P}$ of length $n$ and given $k \leq n$, there exists a condition $q$ such that $q \leq_{k} p$ and $q$ decides $\varphi$ modulo $k$, that is if $r \leq q$ decides $\varphi$ and $q^{\prime}$ is the condition obtained from $q$ by replacing $q \uparrow k$ with $r \uparrow k$, then $q^{\prime}$ decides $\varphi$ the same way $r$ does.

(4) $\mathbb{P}$ preserves $\lambda^{+}$;

(5) forcing with $\mathbb{P}$ turns $\lambda^{+}$into $\aleph_{\omega^{2}+1}$.

We fix a generic filter $G$ for $\mathbb{S}_{0}$ over $V$. In $V[G]$, we define

$$
\mathbb{P}^{*}:=\{p \in \mathbb{P} ; \text { the } S \text {-part of } p \text { is in } G\}
$$

ordered as a subposet of $\mathbb{P}$. We should point out that if the length of $p \in \mathbb{P}$ is $k>1$, then its $S$-part formally does not belong to $\mathbb{S}_{0}$ but to $\mathbb{S}_{k+1}$; however $\mathbb{S}_{k+1}$ naturally embeds into $\mathbb{S}_{0}$ in such a way that $G$ naturally induces an $\mathbb{S}_{k+1}$-generic filter.

Lemma 4.2 (Magidor and Shelah [5, Lemma 6]). $V^{\mathbb{P}} \subseteq V^{\mathbb{S}_{0} * \mathbb{P}^{*}}$.

We will perform the proof of Theorem 1.2 in $V^{\mathbb{S}_{0}}$ where we will work with conditions of $\mathbb{P}^{*}$. The nice feature of $V^{\mathbb{S}_{0}}$ is that in this model $\kappa_{0}$ is still supercompact and, for every $n<\omega$, we can easily get a generic supercompact embedding with critical point $\kappa_{n}$. Moreover, working with conditions of $\mathbb{P}^{*}$ allow us to use the following nice property.

REMARK 4.3. Every two conditions of $\mathbb{P}^{*}$ with the same stem are compatible.

It is convenient to introduce a notation for the $S$-part of a condition $p$ in $\mathbb{P}^{*}$, say $\operatorname{Spart}(p)$. For a condition $p \in \mathbb{P}^{*}$ and for $j \geq \lg (p)$ we denote by $\operatorname{Spart}(p)(j)$ the $\mathrm{j}$-th coordinate of the sequence $\operatorname{Spart}(p)$.

A stem $h$ and a condition $r \in \mathbb{S}_{0}$ determine a unique condition $p \in \mathbb{P}$ that we call the closure of $h$ with $r$ and we denote it $c l(h, r)$. This is the condition $p=\left\langle\alpha_{0}, g_{0}, f_{0}, \ldots, \alpha_{n-1}, g_{n-1}, f_{n-1}, A_{n}, g_{n}, F_{n}, \ldots\right\rangle$ whose stem is $h$ and such that for all $j$ above $n, g_{j}=r(j), A_{j}^{p}=\kappa_{j}$ and $F_{j}^{p}$ is the function that associate to every $\alpha \in A_{j}^{p}=\kappa_{j}$ the maximal condition of $\operatorname{Coll}\left(\alpha^{+\omega+2},<\kappa_{j}\right)$.

Assume $\left\langle g_{n}\right\rangle_{n<\omega}$ and $\left\langle h_{n}\right\rangle_{n<\omega}$ are two conditions in $\mathbb{S}_{0}$, we let

$$
\left\langle g_{n}\right\rangle_{n<\omega} \sim\left\langle h_{n}\right\rangle_{n<\omega}: \Longleftrightarrow \text { for large enough } i, g_{i}=h_{i} .
$$

For $g \in \mathbb{S}_{0}$, we denote by $[g]$ its equivalence class. Define $\mathbb{S}_{0} / \sim:=\left\{[g] ; g \in \mathbb{S}_{0}\right\}$, with the ordering $[g] \leq[h]$ if and only if for large enough $i, g_{i} \leq h_{i}$. Let $G^{*}:=$ $\{[g] ; g \in G\}$, then $V[G]$ is a generic extension of $V\left[G^{*}\right]$ via the forcing $\mathbb{S}_{0} / G^{*}$.

LEMmA 4.4 (Magidor and Shelah [5]). If $H$ is a generic filter for $\mathbb{P}^{*}$ over $V\left[G^{*}\right]$, then $V\left[G^{*}\right][H]=V[H]$. 
Proof. It is enough to show that $G^{*}$ can be defined in $V[H]$. We prove that

$$
G^{*}=\left\{[r] \in \mathbb{S}_{0} / \sim ; \exists p \in H([\operatorname{Spart}(p)] \leq[r])\right\} .
$$

If $p \in H$, then by definition of $\mathbb{P}^{*}$ we have $\operatorname{Spart}(p) \in G$, hence $[\operatorname{Spart}(p)] \in G^{*}$. Conversely, if $[r] \in G^{*}$, then an easy density argument shows that there exists $p \in H$ such that $[\operatorname{Spart}(p)] \leq[r]$. Indeed, if $q \in \mathbb{P}^{*}$, then $[\operatorname{Spart}(q)]$ is in $G^{*}$ and it is therefore compatible with $[r]$; then, one can easily extend $q$ to a condition $p \in \mathbb{P}^{*}$ such that $[\operatorname{Spart}(p)] \leq[r]$.

We now define in $V\left[G^{*}\right]$ the poset

$$
\mathbb{P}^{* *}:=\left\{p \in \mathbb{P} ;[\operatorname{Spart}(p)] \in G^{*}\right\} .
$$

Proposition 4.5. In $V\left[G^{*}\right]$, the product $\mathbb{P}^{* *} \times \mathbb{S}_{0} / G^{*} \times \mathbb{S}_{0} / G^{*}$ has the $\lambda^{+}$-chain condition.

Proof. Assume that for some $r \in \mathbb{S}_{0}$,

$$
[r] \Vdash \dot{A} \subseteq \mathbb{P}^{* *} \times \mathbb{S}_{0} / G^{*} \times \mathbb{S}_{0} / G^{*} \text { is a maximal antichain. }
$$

For every $m<\omega$, we define two sets $\mathbb{Q}_{m}$ and $H_{m}$ as follows:

- $\mathbb{Q}_{m}:=\prod_{i<m} \operatorname{Coll}\left(\kappa_{i}^{+2},<\kappa_{i+1}\right)$.

- $H_{m}$ is the set of all sequences of the form

$$
\left(\alpha_{0}, g_{0}, f_{0}, \ldots, \alpha_{k-1}, g_{k-1}, f_{k-1}, g_{k}, \ldots, g_{m-1}\right),
$$

where $k \leq m$ and there is $p \in \mathbb{P}$ of length $k$ with stem

$$
\left(\alpha_{0}, g_{0}, f_{0}, \ldots, \alpha_{k-1}, g_{k-1}, f_{k-1}\right)
$$

such that $g_{i}=g_{i}^{p}$ for $k \leq i<m$.

For a condition $p \in \mathbb{P}$ of length $k \leq m$, we denote by $h_{m}(p)$ the sequence

$$
\left(\alpha_{0}^{p}, g_{0}^{p}, f_{0}^{p}, \ldots, \alpha_{k-1}^{p}, g_{k-1}^{p}, f_{k-1}^{p}, g_{k}^{p}, \ldots, g_{m-1}^{p}\right) .
$$

Note that $h_{m}(p)$ belongs to $H_{m}$.

As every $H_{m} \times \mathbb{Q}_{m} \times \mathbb{Q}_{m}$ has size $\kappa_{m}$, the union $\bigcup_{m<\omega} H_{m} \times \mathbb{Q}_{m} \times \mathbb{Q}_{m}$ has $\lambda$ many elements and we can enumerate this poset as $\left\{\left(h_{\beta}, s_{\beta}^{0}, s_{\beta}^{1}\right) ; \beta<\lambda\right\}$, where for $\kappa_{i}<\beta<\kappa_{i+1},\left(h_{\beta}, s_{\beta}^{0}, s_{\beta}^{1}\right)$ belongs to $H_{i+1} \times \mathbb{Q}_{i+1} \times \mathbb{Q}_{i+1}$. For $q \in \mathbb{S}_{0}$ and $p \in \mathbb{Q}_{m}$, we denote by $q * p$ the unique condition $u \in \mathbb{S}_{0}$ such that $u \uparrow m=p$ and $u(i)=q(i)$, for every $i \geq m$. We inductively define a decreasing sequence $\left\langle r_{\beta} ; \beta<\lambda\right\rangle$ of conditions in $\mathbb{S}_{0}$, such that for all $\beta, \gamma$ between $\kappa_{i}$ and $\kappa_{i+1}$, we have $r_{\beta}\left\lceil i+1=r_{\gamma}\lceil i+1\right.$.

We let $r_{0}:=r$. For $\beta$ limit, $r_{\beta}$ is defined by $r_{\beta}(i):=\bigcup_{\gamma<\beta} r_{\gamma}(i)$, for every $i$ (the inductive hypothesis and the closure of $\operatorname{Coll}\left(\kappa_{i}^{+2},<\kappa_{i+1}\right)$, for $\kappa_{i}>\beta$, guarantee that $r_{\beta}$ is a condition in $\mathbb{S}_{0}$ ).

Suppose that $r_{\beta}$ has been defined, we want to define $r_{\beta+1}$. Let $m$ be the least such that $\beta<\kappa_{m}$, and let $\varphi_{\beta}$ be the following statement:

"There exists $\left(p, q^{0}, q^{1}\right) \in \dot{A}$ such that

(1) $h_{m}(p)=h_{\beta}$ (hence $p$ is a condition of length $m$ ),

(2) $q^{0} \uparrow m=s_{\beta}^{0}$ and $q^{1} \uparrow m=s_{\beta}^{1}$,

(3) for $j \geq m$ the conditions $q^{0}(j), q^{1}(j), r_{\beta}(j)$ and $g_{j}^{p}$ (the $j$-th coordinate of the $S$-part of $p$ ) are pairwise compatible". 
There exists a condition $r_{\beta+1}$ such that $\left[r_{\beta+1}\right] \leq\left[r_{\beta}\right]$ and $\left[r_{\beta+1}\right]$ decides the statement $\varphi_{\beta}$. We can assume without loss of generality that $r_{\beta+1}\left\lceil m+1=r_{\beta}\lceil m+1\right.$ or we replace $r_{\beta+1}$ by an equivalent condition. So the inductive hypothesis is satisfied.

If $\left[r_{\beta+1}\right] \Vdash \varphi_{\beta}$, then let $\left(p_{\beta}, q_{\beta}^{0}, q_{\beta}^{1}\right)$ witness it. The condition $\left[r_{\beta+1}\right]$ forces that $\left[q_{\beta}^{0}\right],\left[q_{\beta}^{1}\right],\left[r_{\beta}\right]$ and the class of the $S$-part of $p_{\beta}$ are in $G^{*}$, hence $\left[r_{\beta+1}\right]$ must be stronger than all of them. It follows that for all $j$ above some $k$, we have

$$
r_{\beta+1}(j) \leq q_{\beta}^{0}(j), q_{\beta}^{1}(j), r_{\beta}(j), g_{j}^{p_{\beta}} .
$$

If $m<k$, then for $j$ between $m$ and $k$ the conditions $q_{\beta}^{0}(j), q_{\beta}^{1}(j), r_{\beta}(j)$, and $g_{j}^{p_{\beta}}$ are compatible by item 3 , hence $q_{\beta}^{0}(j) \cup q_{\beta}^{1}(j) \cup r_{\beta}(j) \cup g_{j}^{p_{\beta}}$ is a condition in $\operatorname{Coll}\left(\kappa_{i}^{++},<\kappa_{i+1}\right)$. Since we can replace $r_{\beta+1}$ by an equivalent condition, we can assume without loss of generality that for all $j$ between $m$ and $k$, we have $r_{\beta+1}(j)=q_{\beta}^{0}(j) \cup q_{\beta}^{1}(j) \cup r_{\beta}(j) \cup g_{j}^{p_{\beta}}$. It follows that

(1) $r_{\beta+1} * s_{\beta}^{0} \leq q_{\beta}^{0}$,

(2) $r_{\beta+1} * s_{\beta}^{1} \leq q_{\beta}^{1}$,

(3) for all $j \geq m, r_{\beta+1}(j) \leq g_{j}^{p_{\beta}}$.

Now, we let $r_{\infty} \in \mathbb{S}_{0}$ be defined by $r_{\infty}(i):=\bigcup_{\beta<\lambda} r_{\beta}(i)$. We define

$$
E:=\left\{\left(p_{\beta}, q_{\beta}^{0}, q_{\beta}^{1}\right) ; \beta<\lambda,\left[r_{\beta+1}\right] \Vdash \varphi_{\beta}\right\} .
$$

We show that $\left[r_{\infty}\right]$ forces that every element of $\dot{A}$ is compatible with an element of $E$. Since $E$ has size $\lambda$, this will prove that the size of the antichain is at most $\lambda$.

Assume that for some $s \in \mathbb{S}_{0}$ and for some $\left(p, q^{0}, q^{1}\right)$ we have $[s] \leq\left[r_{\infty}\right]$ and $[s]$ forces that $\left(p, q^{0}, q^{1}\right) \in \dot{A}$. Without loss of generality $s \leq r_{\infty}$. Also [s] forces that $\left[q^{0}\right],\left[q^{1}\right]$ and the class of the $S$-part of $p$ are in $G^{*}$, hence $[s]$ is stronger than all those conditions. For some $m$, we have $s(i) \supseteq q^{0}(i) \cup q^{1}(i) \cup g_{i}^{p}$ for every $i \geq m$. The triple $\left(h_{m}(p), q^{0} \uparrow m, q^{1} \uparrow m\right)$ appears in our enumeration as $\left(h_{\beta}, s_{\beta}^{0}, s_{\beta}^{1}\right)$ for some $\beta<\kappa_{m}$. Clearly $\left(p, q^{0}, q^{1}\right)$ witnesses the truth of $\varphi_{\beta}$ thus $[s] \Vdash \varphi_{\beta}$ because $[s] \leq\left[r_{\beta+1}\right]$. So $[s]$ forces that both $\left(p, q^{0}, q^{1}\right)$ and $\left(p_{\beta}, q_{\beta}^{0}, q_{\beta}^{1}\right)$ are in the antichain. We prove that they are compatible, hence they are equal. The conditions $p$ and $p_{\beta}$ have the same stem, let $i$ be their common length. We claim that $g_{i}^{p}$ and $g_{i}^{p_{\beta}}$ are compatible. For $i<m$ this is true because $s(i)$ extends both $g_{i}^{p}$ and $g_{i}^{p_{\beta}}$. Similarly $q^{0}$ and $q^{1}$ are compatible with $q_{\beta}^{0}$ and $q_{\beta}^{1}$ respectively. This completes the proof.

Corollary 4.6. $\vdash_{\mathbb{P}^{* *}} \mathbb{S}_{0} / G^{*} \times \mathbb{S}_{0} / G^{*}$ is $\lambda^{+}$-c.c.

Proof. Suppose for a contradiction that for some $p \in \mathbb{P}^{* *}$, we have

$$
p \Vdash \dot{A} \text { is an antichain of size } \lambda^{+} \text {. }
$$

For every $\alpha<\lambda^{+}$, we fix a condition $p_{\alpha} \leq p$ that decides the value of the $\alpha$-th element of $\dot{A}$ as a pair $\left(s_{\alpha}^{0}, s_{\alpha}^{1}\right) \in \mathbb{S}_{0} / G^{*} \times \mathbb{S}_{0} / G^{*}$. For $\alpha<\beta<\lambda^{+}$, if $\left(q, t^{0}, t^{1}\right) \leq$ $\left(p_{\alpha}, s_{\alpha}^{0}, s_{\alpha}^{1}\right),\left(p_{\beta}, s_{\beta}^{0}, s_{\beta}^{1}\right)$, then

$$
q \Vdash\left(s_{\alpha}^{0}, s_{\alpha}^{1}\right),\left(s_{\beta}^{0}, s_{\beta}^{1}\right) \in \dot{A} \text { and }\left(t_{0}, t^{1}\right) \leq\left(s_{\alpha}^{0}, s_{\alpha}^{1}\right),\left(s_{\beta}^{0}, s_{\beta}^{1}\right)
$$

contradicting the fact that $\dot{A}$ is an antichain. It follows that $\left\{\left(p_{\alpha}, s_{\alpha}^{0}, s_{\alpha}^{1}\right) ; \alpha<\lambda^{+}\right\}$ is an antichain of $\mathbb{P}^{*} \times \mathbb{S}_{0} / G^{*} \times \mathbb{S}_{0} / G^{*}$. By Proposition 4.5 this product has the $\lambda^{+}$-chain condition, so we have a contradiction. 
$\S 5$. Forcing the tree property at $\aleph_{\omega^{2}+1}$ with $\mathbb{P}$. In this section, we prove that there exists a $\mathbb{P}$-generic extension of $V$ in which $\aleph_{\omega^{2}+1}$ has the tree property, thus we demonstrate Theorem 1.2. In the proof we will use a technique described in Sinapova's paper [10] for getting the tree property at the successor of a singular from a diagonal Prikry-type forcing. However, Sinapova's approach is based on a typical property of diagonal Prikry-type forcings, namely that any two conditions with the same stem are compatible; this is not true for the forcing $\mathbb{P}$. The forcing $\mathbb{P}^{*}$ on the contrary does satisfy this property, so we will work with $\mathbb{P}^{*}$ over $V[G]$ and make the relevant changes to apply Sinapova's technique to our case.

Suppose for a contradiction that no $\mathbb{P}$-generic extension of $V$ forces the tree property at $\lambda^{+}$, then we can find a $\mathbb{P}$-name $\dot{T}$ such that

$$
\emptyset \Vdash_{\mathbb{P}} \dot{T} \text { is a } \lambda^{+} \text {-Aronszajn tree. }
$$

We can assume that $\dot{T}$ is a name for a subset of $\lambda^{+} \times \lambda$.

We are going to prove that in $V[G]$ there exists a sequence of pairwise compatible conditions $\left\langle p_{\beta} ; \beta \in J\right\rangle$ in $\mathbb{P}^{*}$ and a sequence of elements $\left\langle u_{\beta} ; \beta \in J\right\rangle$ in $\lambda^{+} \times \lambda$, where $J$ is a cofinal subset of $\lambda^{+}$, such that for all $\beta<\beta^{\prime}$ in $J$ the weakest common extension of $p_{\beta}$ and $p_{\beta^{\prime}}$ forces $u_{\beta}<_{\dot{T}} u_{\beta^{\prime}}$. Once those sequences are defined, we get a contradiction with the following argument. We claim that there exists a generic filter $H$ for $\mathbb{P}^{*}$ over $V[G]$ such that $\left\{\beta ; p_{\beta} \in H\right\}$ is cofinal in $\lambda^{+}$. Indeed, if no such filter exists, then we would have $\emptyset \Vdash_{\mathbb{P}^{*}}\left\{\beta ; p_{\beta} \in \dot{G}\right\}$ is bounded. As $\mathbb{P}^{*}$ is $\lambda^{+}$-c.c. there would be $\delta<\lambda^{+}$such that $\emptyset \Vdash_{\mathbb{P}^{*}}\left\{\beta ; p_{\beta} \in \dot{G}\right\} \subseteq \delta$. Let $\delta^{\prime} \in J$ above $\delta$, then $p_{\delta^{\prime}} \Vdash p_{\delta^{\prime}} \notin \dot{G}$, a contradiction. It follows that $B:=\left\{u_{\beta} ; p_{\beta} \in H\right\}$ is a cofinal branch for the tree in $V[G][H]$. We recall that $H$ was $\mathbb{P}^{*}$-generic over $V[G]$, it is also $\mathbb{P}^{* *}$-generic over $V\left[G^{*}\right]$. We have $V[G][H]=V\left[G^{*}\right][H]\left[G / G^{*}\right]=V[H]\left[G / G^{*}\right]$, namely $V[G][H]$ can be seen as a generic extension of $V[H]$ via the forcing $\mathbb{S}_{0} / G^{*}$. By Corollary 4.6, we know that $\mathbb{S}_{0} / G^{*} \times \mathbb{S}_{0} / G^{*}$ is $\lambda^{+}$-c.c. in $V[H]$. Since $B$ is approximated, we can apply Lemma 2.1 , thus $B$ exists in $V[H]$. So we found a cofinal branch for $\dot{T}$ in a $\mathbb{P}$-generic extension of $V$, contradicting the assumption $\emptyset \vdash_{\mathbb{P}} \dot{T}$ is Aronszajn.

The first step is to prove the following.

Lemma 5.1. In $V[G]$ there are $n, m<\omega$ and a cofinal set $I \subseteq \lambda^{+}$such that for all $\alpha<\beta$ in I, one can find $\zeta, \eta<\kappa_{m}$ and a condition $q \in \mathbb{P}^{*}$ of length $n$ such that $q \Vdash(\alpha, \zeta)<_{\dot{T}}(\beta, \eta)$.

Proof. We let $j: V[G] \rightarrow M$ be the elementary embedding corresponding to $\dot{F}_{0}^{G}$. So the critical point of $j$ is $\kappa_{0}, j\left(\kappa_{0}\right)>\lambda^{+}$and $M$ is closed by sequences of length $\lambda^{+}$. We fix $\bar{H}$ a generic filter for $j\left(\mathbb{P}^{*}\right)$ over $V[G]$ such that the first element of the $\alpha$-sequence added by $\bar{H}$ is $\kappa_{0}$. This implies that $\lambda^{+}$is not collapsed, because $\lambda^{+}=\kappa_{0}^{+\omega+1}$ and, by definition of our forcing, $\bar{H}$ does not collapse any cardinal between $\kappa_{0}^{+\omega}$ and $\kappa_{0}^{+\omega+2}$. Fix any ordinal $\gamma$ between sup $j^{\prime \prime} \lambda^{+}$and $j\left(\lambda^{+}\right)$. Let $T^{*}:=j(\dot{T})^{\bar{H}} . T^{*}$ is a $j\left(\lambda^{+}\right)$-tree, so we can fix a node $u$ of $T^{*}$ on level $\gamma$. For every $\beta<\lambda^{+}$, there are $m_{\beta}<\omega, \zeta_{\beta}<j\left(\kappa_{m_{\beta}}\right)$ and a condition $p_{\beta} \in \bar{H}$ such that

$$
p_{\beta} \Vdash_{j(\mathbb{P} *)}\left(j(\beta), \zeta_{\beta}\right)<_{j(\dot{T})} u .
$$


For some $n, m<\omega$ and for a cofinal $I^{*} \subseteq \lambda^{+}$, we have $\lg \left(p_{\beta}\right)=n$ and $m_{\beta}=m$, for every $\beta \in I^{*}$. We can write each condition $p_{\beta}$ with $\beta \in I^{*}$ as

$$
p_{\beta}=\left\langle\kappa_{0}, g_{0}^{\beta}, f_{0}^{\beta}, \ldots, \alpha_{n-1}^{\beta}, g_{n-1}^{\beta}, f_{n-1}^{\beta}, A_{n}^{\beta}, g_{n}^{\beta}, F_{n}^{\beta}, \ldots\right\rangle .
$$

As the $p_{\beta}$ 's are pairwise compatible conditions, they satisfy the following properties:

(1) the $p_{\beta}$ 's have the same $\alpha$-part $\left\langle\alpha_{0}, \ldots, \alpha_{n-1}\right\rangle$;

(2) we can assume that for some $g_{0} \in \operatorname{Coll}\left(\omega, \kappa_{0}^{+\omega}\right)$, we have $g_{0}^{\beta}=g_{0}$ for all $\beta^{*}$ (we can shrink $I^{*}$ if necessary);

(3) for every $0<i<n$, the sequence $\left\langle g_{i}^{\beta}\right\rangle_{\beta \in I}$ has a lower bound $g_{i}$ (indeed $\operatorname{Coll}\left(j\left(\kappa_{i-1}\right),<\alpha_{i}\right)$ has closure $\left.>\lambda^{+}\right)$;

(4) for $i<n$, the sequence $\left\langle f_{i}^{\beta}\right\rangle_{\beta \in I}$ has a lower bound $f_{i}$ (these are conditions in $\operatorname{Coll}\left(\alpha_{i}^{+\omega+2}, j\left(\kappa_{i}\right)\right)$ which has closure $\left.>\kappa_{0}^{+\omega+1}=\lambda^{+}\right)$.

So we defined a stem

$$
s:=\left\langle\kappa_{0}, g_{0}, f_{0}, \ldots, \alpha_{n-1}, g_{n-1}, f_{n-1}\right\rangle
$$

such that for all $\beta \in I^{*}$, there exists a condition $p \in j\left(\mathbb{P}^{*}\right)$ with stem $s$ forcing $\left(j(\beta), \zeta_{\beta}\right)<_{T *} u$. By letting

$$
I:=\left\{\beta<\lambda^{+} ; \exists p \in j\left(\mathbb{P}^{*}\right)\left(\operatorname{stem}(p)=s \wedge \exists \zeta<j\left(\kappa_{m}\right)(p \Vdash(j(\beta), \zeta)<u)\right)\right\}
$$

we get a cofinal subset of $\lambda^{+}$which is in $V[G]$ and has the desired property. Indeed, if $\beta<\beta^{\prime}$ are in $I$, then there are $p, p^{\prime} \in j\left(\mathbb{P}^{*}\right)$ with stem $s$ and $\zeta, \eta<j\left(\kappa_{m}\right)$ such that

(1) $p \Vdash(j(\beta), \zeta)<_{j(\dot{T})} u$,

(2) $p^{\prime} \Vdash\left(j\left(\beta^{\prime}\right), \eta\right)<_{j(\dot{T})} u$.

As they have the same stem, the two conditions are compatible, so there exists a condition $q^{*} \leq p, p^{\prime}$ forcing $(j(\beta), \zeta)<_{j(\dot{T})} u$ and $\left(j\left(\beta^{\prime}\right), \eta\right)<_{j(\dot{T})} u$.

It follows that $q^{*} \Vdash(j(\beta), \zeta)<_{j(\dot{T})}\left(j\left(\beta^{\prime}\right), \eta\right)$. By elementarily, we can find a condition $q \in \mathbb{P}^{*}$ of length $n$ and two ordinals $\zeta, \eta<\kappa_{m}$ such that $q \Vdash_{\mathbb{P}^{*}}^{V[G]}$ $(\beta, \zeta)<_{\dot{T}}\left(\beta^{\prime}, \eta\right)$. That completes the proof of the lemma.

We fix $n, m$, and $I$ as in the conclusion of the above lemma, without loss of generality $n \leq m$. In $V[G]$ we say that a stem $s$ 'forces' a statement $\varphi$ and we write $s \Vdash \varphi$, when there is a condition $p \in \mathbb{P}^{*}$ with stem $s$ such that $p \Vdash \varphi$. We prove the following.

Lemma 5.2. In $V[G]$, there exists a cofinal $J \subseteq I$, a stem $h$ of $\mathbb{P}^{*}$ length $n$ and $a$ sequence $\left\langle u_{\beta} ; \beta \in J\right\rangle$ with $u_{\beta} \in\{\beta\} \times \kappa_{m}$ for $\beta \in J$, such that for every $\beta<\beta^{\prime}$ in $J$, we have $h \Vdash u_{\beta}<_{T} u_{\beta^{\prime}}$.

Proof. Let $l=m+2$. If $V_{l}$ is the $\mathbb{S}_{l}$-generic extension determined by $G$, then $\kappa_{l}$ is supercompact in $V_{l}$ and there is a $\lambda^{+}$-supercompact elementary embedding $j: V_{l} \rightarrow M_{l}$ with critical point $\kappa_{l}$. Let $G_{C}$ be the generic filter for $\operatorname{Coll}\left(\kappa_{0}^{+2},<\kappa_{1}\right) \times$ $\cdots \times \operatorname{Coll}\left(\kappa_{l-1}^{+2},<\kappa_{l}\right)$ determined by $G$, then by forcing with $\mathbb{C}_{\text {Tail }}:=\operatorname{Coll}\left(\kappa_{l-1}^{+2}\right.$, $\left.<j\left(\kappa_{l}\right) \backslash \kappa_{l}\right)$ over $V[G]$ we get a generic object $H^{*}$ such that $j[G] \subseteq G_{C} * H^{*}$, hence we can lift $j$ to an embedding $j^{*}: V[G] \rightarrow M_{l}\left[G_{C}\right]\left[H^{*}\right]$ that we rename $j$. 
Let $\gamma \in j(I)$ be above sup $j^{\prime \prime} \lambda^{+}$. For every $\beta \in I$, we fix $\zeta_{\beta}, \eta_{\beta}<\kappa_{m}$ and a condition $p_{\beta} \in j\left(\mathbb{P}^{*}\right)$ of length $n$ with stem $h_{\beta}$ such that

$$
p_{\beta} \Vdash\left(j(\beta), \zeta_{\beta}\right)<_{j(\dot{T})}\left(\gamma, \eta_{\beta}\right) .
$$

In $V[G]$ we define a system $\left\{R_{h} ; h\right.$ is a stem $\}$ over $\lambda^{+} \times \kappa_{m}$ by letting

$$
R_{h}:=\left\{\langle u, v\rangle ; \exists p \in \mathbb{P}, \operatorname{stem}(p)=h, p \Vdash u<_{\dot{T}} v\right\} .
$$

Every $R_{h}$ is transitive, because in $V[G]$ two conditions of $\mathbb{P}$ with the same stem are compatible. We also define for every stem $h$ and for every $\eta<\kappa_{m}$ a set

$$
b_{h, \eta}:=\left\{(\beta, \zeta) \in \lambda^{+} \times \kappa_{m} ; \exists p \in j(\mathbb{P})\left(\operatorname{stem}(p)=h \wedge p \Vdash(j(\beta), \zeta)<_{j(\dot{T})}(\gamma, \eta)\right\} .\right.
$$

Note that every $b_{h, \delta}$ is an $R_{h}$-branch. Moreover, in $V[G] \lambda^{+}$is regular and the stems of $j(\mathbb{P})$ of length $n$ are $<\kappa_{n}$. The forcing $\mathbb{C}_{\text {Tail }}$ is $\kappa_{l-1}^{+2}$-closed, hence it doesn't add $<\kappa_{n}$-sequences. So we can find in $V[G]\left[H^{*}\right]$ a cofinal $J \subseteq I$, a stem $h^{*}$ and two ordinals $\zeta^{*}, \eta^{*}$ such that for $\beta$ in $J$, we have $h_{\beta}=h^{*}, \zeta_{\beta}=\zeta^{*}$ and $\eta_{\beta}=\eta^{*}$. Thus $b_{h^{*}, \eta^{*}}$ is a cofinal $R_{h^{*}}$-branch and we can apply Theorem 2.5 . We get that the system has a cofinal branch in $V[G]$, i.e., for some stem $h$, there exists a cofinal $J \subseteq I$, and a sequence $\left\langle\sigma_{\beta} ; \beta \in J\right\rangle$ such that for $\beta<\beta^{\prime}$ in $J, h \Vdash\left(\beta, \sigma_{\beta}\right)<_{\dot{T}}\left(\beta^{\prime}, \sigma_{\beta^{\prime}}\right)$. Set $u_{\beta}:=\left(\beta, \sigma_{\beta}\right)$ for $\beta \in J$, then $J$ and $\left\langle u_{\beta} ; \beta \in J\right\rangle$ are as required.

Let $h, J$, and $\alpha \mapsto u_{\alpha}$ be as in the conclusion of the above lemma. By shrinking $J$, we may assume that for some $\zeta<\kappa_{m}$, we have $u_{\alpha}=(\alpha, \zeta)$ for each $\alpha \in J$.

Lemma 5.3. Suppose that $s$ is a stem of length $k, L \subseteq \lambda^{+}$is unbounded and for all $\alpha<\beta$ with $\alpha, \beta \in L, s \Vdash u_{\alpha}<_{T} u_{\beta}$. Then, there are $\rho<\lambda^{+}$and sets $\left\langle A^{\alpha}, g^{\alpha}, F^{\alpha}\right\rangle_{\alpha \in L \backslash \rho}$ in $V[G]$ such that:

(1) $A^{\alpha}$ is in $U_{k}, g^{\alpha}$ is in the generic filter for $\operatorname{Coll}\left(\kappa_{k-1}^{++},<\min \left(A^{\alpha}\right)\right)$ induced by $G, F^{\alpha}$ is a function of domain $A^{\alpha}$ such that for every $x \in A^{\alpha}, F^{\alpha}(x) \in$ $\operatorname{Coll}\left(\kappa_{x}^{+\omega+2},<\kappa_{k}\right)$ and $\left[F^{\alpha}\right]_{U_{k}} \in K_{k} ;$

(2) for every $\alpha<\beta$ in $L \backslash \rho$, for all $x \in A^{\alpha} \cap A^{\beta}$,

$$
s \frown\left\langle x, g^{\alpha} \cup g^{\beta}, F^{\alpha}(x) \cup F^{\beta}(x)\right\rangle \Vdash u_{\alpha}<_{\dot{T}} u_{\beta} .
$$

Proof. Let $l$ be $k+3$. If $V_{l}$ is the $\mathbb{S}_{l}$-generic extension determined by $G$, then $\kappa_{l}$ is supercompact in $V_{l}$ and there is a $\lambda^{+}$-supercompact elementary embedding $j: V_{l} \rightarrow M_{l}$ with critical point $\kappa_{l}$. Let $G_{C}$ be the generic filter for $\operatorname{Coll}\left(\kappa_{0}^{+2},<\right.$ $\left.\kappa_{1}\right) \times \cdots \times \operatorname{Coll}\left(\kappa_{l-1}^{+2},<\kappa_{l}\right)$ determined by $G$, then forcing with $\mathbb{C}_{\text {tail }}:=\operatorname{Coll}\left(\kappa_{l-1}^{+2},<\right.$ $\left.j\left(\kappa_{l}\right) \backslash \kappa_{l}\right)$ over $V[G]$ we get a generic object $H^{*}$ such that $j[G] \subseteq G_{C} * H^{*}$, hence we can lift $j$ to an embedding $j^{*}: V[G] \rightarrow M_{l}\left[G_{C}\right]\left[H^{*}\right]$ that we rename $j$. Note that the forcing $\mathbb{C}_{\text {tail }}$ is $\kappa_{l-1}$-closed, that is $\kappa_{k+2}$-closed. Choose $\gamma \in j(L)$ above $j\left[\lambda^{+}\right]$. By elementarily, we can pick for all $\alpha \in L$ a condition $p_{\alpha} \in j\left(\mathbb{P}^{*}\right)$ with stem $s$ such that

$$
p_{\alpha} \Vdash(j(\alpha), \zeta)<_{j(\dot{T})}(\gamma, \zeta) .
$$

Every $p_{\alpha}$ is of the form $s \frown\left(A_{k}^{\alpha}, g_{k}^{\alpha}, F_{k}^{\alpha}, \ldots\right)$ where $A_{k}^{\alpha} \in j\left(U_{k}\right)=U_{k} \subseteq \mathcal{P}\left(\kappa_{k}\right)$, $g_{k}^{\alpha}$ is in the generic filter for $\operatorname{Coll}\left(\kappa_{k-1}^{++},<\min \left(A_{k}^{\alpha}\right)\right)$ induced by $G$, and $F_{k}^{\alpha}$ is a function with domain $A_{k}^{\alpha}$ such that for every $\beta \in A_{k}^{\alpha}, F_{k}^{\alpha}(\beta)$ is a condition in $\operatorname{Coll}\left(\beta^{+\omega+2},<\kappa_{k}\right)$ and $\left[F_{k}^{\alpha}\right]_{U_{k}} \in K_{k}$ There are $\left|\mathcal{P}\left(\kappa_{k}\right)\right|=\kappa_{k+1}$-many possible triples $(A, g, F)$ in the range of the function $\alpha \mapsto\left(A_{k}^{\alpha}, g_{k}^{\alpha}, F_{k}^{\alpha}\right)$. Since $\lambda^{+}$is regular 
in $V[G]\left[H^{*}\right]$ and $\mathbb{C}_{\text {tail }}$ adds no sequences of length less than $\kappa_{k+2}$, the function $\alpha \mapsto\left(A_{k}^{\alpha}, g_{k}^{\alpha}, F_{k}^{\alpha}\right)$ must be constant on an unbounded subset $L^{\prime}$ of $L$. So there is $\left(A^{*}, g^{*}, F^{*}\right)$ such that $\left(A^{*}, g^{*}, F^{*}\right)=\left(A_{k}^{\alpha}, g_{k}^{\alpha}, F_{k}^{\alpha}\right)$ for every $\alpha \in L^{\prime}$.

Now we step back to $V[G]$ where we define $E$ as the set of all triples $(A, g, F)$ such that $A \in U_{k}, g$ is in the generic filter for $\operatorname{Coll}\left(\kappa_{k-1}^{++},<\min (A)\right)$ derived by $G$, and $F$ is a function with domain $A$ such that $[F]_{U_{k}} \in K_{k}$ and $F(x) \in \operatorname{Coll}\left(x^{+\omega+2},<\kappa_{k}\right)$ for all $x \in A$. For every $(A, g, F) \in E$, we define a relation $R_{A, g, F}$ on $L \times\{\zeta\}$, by letting $a R_{A, g, F} b$ when there exists a condition $p$ such that $p \nmid k+1=s \frown(A, g, F)$ and $p \Vdash a<_{\dot{T}} b$. Then $\left\{R_{A, g, F}\right\}_{(A, g, F) \in E}$ is a system, because for every two conditions $p, p^{\prime} \in \mathbb{P}^{*}$ extending $s \frown(A, g, F)$ and forcing a statement $\varphi$, we can find a third condition $q \leq p, p^{\prime}$ extending $s \frown(A, g, F)$ and forcing the same statement $\varphi$.

Now in $V[G]\left[H^{*}\right]$ we can define a system of branches for $\left\{R_{A, g, F}\right\}_{(A, g, F) \in E}$ as follows. We let $b_{A, g, F}$ be the set of all pairs $(\alpha, \zeta)$ such that there is a condition $p$ in $j(\mathbb{P})$ such that $p\left\lceil k+1=s \frown(A, g, F)\right.$ and $\left.p \Vdash(j(\alpha), \zeta)<_{j(\dot{T})}(\gamma, \zeta)\right)$ The triple $\left(A^{*}, g^{*}, F^{*}\right)$ defined above determines a cofinal branch $b_{A^{*}, g^{*}, F^{*}}$. By Theorem 2.5 (applied to $\mathbb{C}_{\text {tail }}$ which is $\kappa_{k+2}$-closed), a cofinal branch for the system exists also in $V[G]$. So there exists $L^{*} \subseteq L$ and $(A, g, F)$ such that for all $\alpha<\beta$ in $L^{*}$, there exists a condition $p \in \mathbb{P}^{*}$ extending $s \frown(A, g, F)$ that forces $u_{\alpha}<_{T} u_{\beta}$.

Let $\rho$ be the least element of $L^{*}$ and, for every $\alpha \in(L \backslash \rho) \backslash L^{*}$, let $\alpha^{*}$ be the least element of $L^{*}$ above $\alpha$. For $\alpha \in(L \backslash \rho) \backslash L^{*}$ there exists $\left(\bar{A}^{\alpha}, \bar{g}^{\alpha}, \bar{F}^{\alpha}\right)$ such that some condition extending $s \frown\left(\bar{A}^{\alpha}, \bar{g}^{\alpha}, \bar{F}^{\alpha}\right)$ forces $u_{\alpha}<_{\dot{T}} u_{\alpha^{*}}$. So given $\alpha \in L \backslash \rho$, we define $\left(A^{\alpha}, g^{\alpha}, F^{\alpha}\right)$ as follows. If $\alpha \in L^{*}$, then we let $\left(A^{\alpha}, g^{\alpha}, F^{\alpha}\right)$ be $(A, g, F)$. If $\alpha \notin L^{*}$, then we let $A^{\alpha}$ be a subset of $A \cap \bar{A}^{\alpha}$ such that for every $x \in A^{\alpha}$, $\bar{F}^{\alpha}(x)$ and $F(x)$ are compatible; we let $g^{\alpha}$ be a condition in the generic filter for $\operatorname{Coll}\left(\kappa_{k-1}^{++},<\min \left(A \cap \bar{A}^{\alpha}\right)\right)$ induced by $G$, and we let $F^{\alpha}(x)=\bar{F}^{\alpha}(x) \cup F(x)$. The sequence $\left\langle A^{\alpha}, g^{\alpha}, F^{\alpha}\right\rangle_{\alpha \in L \backslash \rho}$ is as required.

We are now ready for the final step.

LEMMA 5.4. In $V[G]$ there exists a sequence of pairwise compatible conditions $\left\langle p_{\beta} ; \beta \in J \backslash \rho\right\rangle$ in $\mathbb{P}^{*}$ with stem $h$ where $\rho<\lambda^{+}$and for all $\beta<\beta^{\prime}$ in $J$ the weakest common extension of $p_{\beta}$ and $p_{\beta^{\prime}}$ forces in $\mathbb{P}^{*}$ that $u_{\beta}<_{\dot{T}} u_{\beta^{\prime}}$.

Proof. By induction on $k<\omega$, we define $\left\langle\rho_{k} ; k \geq n\right\rangle$, and $\left\langle A_{k}^{\alpha}, g_{k}^{\alpha}, F_{k}^{\alpha} ; k \geq n\right.$, $\left.\alpha \in J \backslash \rho_{k}\right\rangle$ such that:

(1) for all $\alpha \in J \backslash \rho_{k}$, we have $A_{k}^{\alpha} \in U_{k}, g_{k}$ is in the generic filter for $\operatorname{Coll}\left(\kappa_{k-1}^{++},<\right.$ $\left.\min \left(A_{k}^{\alpha}\right)\right)$ induced by $G$, and $F_{k}^{\alpha}$ is a function with domain $A_{k}^{\alpha}$ with $\left[F_{k}^{\alpha}\right]_{U_{k}} \in$ $K_{k}$ such that $F_{k}^{\alpha}(\beta) \in \operatorname{Coll}\left(\beta^{+\omega+2},<\kappa_{k}\right)$ for every $\beta \in A_{k}^{\alpha}$;

(2) for all $\beta<\beta^{\prime}$ in $J \backslash \rho_{k}$, if $s$ is a stem of the form

$$
h \frown\left\langle\alpha_{n}, g_{n}, f_{n}, \ldots, \alpha_{k}, g_{k}, f_{k}\right\rangle
$$

such that for $n \leq i \leq k, \alpha_{i} \in A_{i}^{\beta} \cap A_{i}^{\beta^{\prime}}, g_{i}$ is in the generic filter for $\operatorname{Coll}\left(\kappa_{i-1}^{++}, \alpha_{i}\right)$ derived from $G$, and $f_{i} \leq F_{i}^{\beta}\left(\alpha_{i}\right) \cup F_{i}^{\beta^{\prime}}\left(\alpha_{i}\right)$, there is a condition of $\mathbb{P}^{*}$ with stem $s$ that forces $u_{\beta}<_{\dot{T}} u_{\beta^{\prime}}$

$\rho_{n}$ and $\left\langle A_{n}^{\alpha}, g_{n}^{\alpha}, F_{n}^{\alpha} ; \alpha \in J \backslash \rho_{n}\right\rangle$ are given by the above lemma applied to $h$. Assume that we have defined $\rho_{k}$, and $\left\langle A_{k}^{\alpha}, g_{k}^{\alpha}, F_{k}^{\alpha} ; \alpha \in J \backslash \rho_{k}\right\rangle$, we want to define $\rho_{k+1}$, and $\left\langle A_{k+1}^{\alpha}, g_{k+1}^{\alpha}, F_{k+1}^{\alpha} ; \alpha \in J \backslash \rho_{k+1}\right\rangle$. For a stem $s=\left\langle\alpha_{0}, g_{0}, f_{0}, \ldots, \alpha_{k}, g_{k}, f_{k}\right\rangle$ 
extending $h$ we let

$$
J^{s}:=\left\{\alpha \in J \backslash \rho_{k} ; \forall i \leq k, \alpha_{i} \in A_{i}^{\alpha}, g_{i} \leq g_{i}^{\alpha} \text { and } f_{i} \leq F_{i}^{\alpha}\left(\alpha_{i}\right)\right\} .
$$

We define $\rho^{s}$ as follows: if $J^{s}$ is bounded in $\lambda^{+}$, then $\rho^{s}$ is a bound; otherwise we let $\rho^{s}$ and $\left\langle A^{s, \alpha}, g^{s, \alpha}, F^{s, \alpha} ; \alpha \in J^{s} \backslash \rho^{s}\right\rangle$ be given by the above lemma applied to $s$ and $J^{s}$. Let

$$
\rho_{k+1}:=\sup \left\{\rho^{s} ; s \text { is a stem of length } k+1 \text { extending } h\right\} .
$$

For each $\alpha \in J \backslash \rho_{n+1}$, let

$$
H_{\alpha}(k+1):=\left\{s ; s \text { is a stem of length } k+1 \text { such that } h \sqsubseteq s \text { and } \alpha \in J^{s}\right\} .
$$

Note that the set $A_{k+1}^{\alpha}:=\bigcap_{s \in H_{\alpha}(k+1)} A^{s, \alpha}$ is in $U_{k+1}$ and $\left[F^{\alpha}\right]_{U_{k+1}}:=$ $\bigcup\left\{\left[F^{s, \alpha}\right]_{U_{k+1}} ; s \in H_{\alpha}(k+1)\right\}$ is in $K_{k+1}$. By shrinking $A_{k+1}^{\alpha}$ we can assume that for all $x \in A_{k+1}^{\alpha}, F^{\alpha}(x)=\bigcup\left\{F^{s, \alpha}(x) ; x>\alpha_{k}\right\}$. We let $g^{\alpha}$ be the condition $\bigcup_{s \in H_{\alpha}(k+1)} g^{s, \alpha}$. We check that (1) and (2) hold for $k+1$. Condition (1) holds by construction. For condition (2), consider $\alpha<\beta$ both in $J \backslash \rho_{k+1}$ and suppose that $s=h \frown\left\langle A_{n}, g_{n}, F_{n}, \ldots, A_{k+1}, g_{k+1}, F_{k+1}\right\rangle$ is a stem of length $k+2$ such that $\alpha, \beta \in J^{s}$. As both $\alpha$ and $\beta$ are in $J^{s}$ and $\rho_{k+1}<\alpha, \beta$, we have that $J^{s}$ is unbounded and $\rho^{s}$ was obtained by applying the previous lemma. Therefore $s \in H_{\alpha}(k+1) \cap H_{\beta}(k+1), \alpha_{k+1} \in A^{s, \alpha} \cap A^{s, \beta}, g_{k+1} \leq g^{s, \alpha}, g^{s, \beta}$ and $f_{k+1} \leq F^{s, \alpha}\left(\alpha_{k+1}\right) \cup F^{s, \beta}\left(\alpha_{k+1}\right)$. So by construction of $A^{s, \alpha}, A^{s, \beta}, F^{s, \alpha}, F^{s, \beta}$ we have $s \Vdash u_{\alpha}<_{\dot{T}} u_{\beta}$.

This completes the definition. Finally, we let $\rho:=\sup _{n<\omega} \rho_{n}$ and for every $\alpha \in$ $J \backslash \rho$, we let $p_{\alpha}$ be the condition $h \frown\left\langle A_{n}^{\alpha}, g_{n}^{\alpha}, F_{n}^{\alpha}, \ldots, A_{j}^{\alpha}, g_{j}^{\alpha}, F_{j}^{\alpha}, \ldots\right\rangle$. Then $\left\langle p_{\alpha} ; \alpha \in J \backslash \rho\right\rangle$ is as desired. Indeed, if $\alpha<\beta$ are in $J \backslash \rho$, then $p_{\alpha}$ and $p_{\beta}$ are compatible in $\mathbb{P}^{*}$ and if $q \leq p_{\alpha}, p_{\beta}$ is in $\mathbb{P}^{*}$, then by construction $\operatorname{stem}(q) \Vdash u_{\alpha}<_{\dot{T}} u_{\beta}$, i.e., there is a condition of $\mathbb{P}^{*}$ with the same stem as $q$ that forces $u_{\alpha}<_{i} u_{\beta}$. Two conditions of $\mathbb{P}^{*}$ with the same stem are compatible, hence $q \forall u_{\alpha} \nless_{\dot{T}} u_{\beta}$. It follows that the weakest common extension of $p_{\alpha}$ and $p_{\beta}$ forces $u_{\alpha}<_{\dot{T}} u_{\beta}$.

That completes the proof of Theorem 1.2.

§6. Stationary set reflection and the failure of the tree property at $\aleph_{\omega^{2}+1}$. In this section we show that the stationary set reflection at $\aleph_{\omega^{2}+1}$ does not imply the tree property. We force from large cardinals a model of $\Delta_{\aleph_{\omega^{2}}, \aleph_{\omega^{2}+1}{ }^{2}}$ where the tree property at $\aleph_{\omega^{2}+1}$ fails, thus we demonstrate Theorem 1.3. The proof combines Magidor and Shelah's technique for getting $\Delta_{\aleph_{\omega^{2}}, \aleph_{\omega^{2}+1}}$ with some ideas from Kunen's paper (see [2]).

We start by assuming the existence of infinitely many supercompact cardinals $\left\langle\kappa_{n}\right\rangle_{n<\omega}$; as usual we assume that the sequence is increasing and that the supercompactness of each $\kappa_{n}$ is indestructible by $\kappa_{n}$-directed closed forcings. We let $\lambda:=\lim _{n<\omega} \kappa_{n}$ and we assume that $2^{\kappa_{n}}=\kappa_{n}^{+}$for every $n<\omega$. We consider the following forcing notion $\mathbb{R}$ : a condition $t$ of $\mathbb{R}$ is either the one-point tree $\{0\}$ or an homogeneous tree $t \subseteq \lambda^{+} 2$ of successor height such that $\langle 0\rangle$ and $\langle 1\rangle$ are in $t$. $\mathbb{R}$ is partially ordered by end-extension. It is proven in Kunen's paper that forcing with $\mathbb{R}$ adds a Suslin tree, let $T$ be such a tree. Kunen observed that the iterated forcing $\mathbb{R} * T$ is equivalent to a $\lambda^{+}$-closed forcing

$$
\mathbb{Q}:=\{(r, t) ; r \in \mathbb{R}, t \in r, \operatorname{ht}(r)=\operatorname{dom}(t)+1, \text { and } r \Vdash t \in \dot{T}\} .
$$


We observe that $\mathbb{Q}$ is even $\lambda^{+}$-directed closed: if $\left\langle r_{\alpha}, t_{\alpha}\right\rangle_{\alpha<\lambda}$ is a directed sequence of conditions in $\mathbb{Q}$, then $r:=\bigcup_{\alpha<\lambda} r_{\alpha}$ is an homogeneous tree of height $\gamma:=$ $\sup _{\alpha<\lambda}$ ht $\left(r_{\alpha}\right)$ and $t:=\bigcup_{\lambda} t_{\alpha}$ is a path through $r$. We can extend $r$ to an homogeneous tree of height $\gamma+1$ by adding at level $\gamma$ all sequences $s \frown(t \backslash \alpha)$ such that $s \in r$, where $t \backslash \alpha$ denotes the unique $s^{\prime}$ such that $t \uparrow \alpha \frown s^{\prime}=t$. This provides a common extension for the sequence $\left\langle r_{\alpha}, t_{\alpha}\right\rangle_{\alpha<\lambda}$.

Since we assumed that the supercompactness of each $\kappa_{n}$ is indestructible by directed closed forcing, $\kappa_{n}$ remains supercompact in $V^{\mathbb{R} * T}$ as well as in the model $V^{(\mathbb{R} * T) \times \mathbb{S}_{n}}\left(\mathbb{S}_{n}\right.$ denotes the same poset as in the previous sections). We use this fact to define in $V$ a variation of the forcing $\mathbb{P}$ discussed in the previous sections: each $\dot{F}_{n}$ is replaced by an $(\mathbb{R} * T) \times \mathbb{S}_{n}$-name for a normal ultrafilter on $\mathcal{P}_{\kappa_{n}}\left(\lambda^{+}\right)$, and $U_{n}$ denotes now the projection of such ultrafilter to a normal utrafilter on $\kappa_{n}$. Since $2^{\kappa_{n}}=\kappa_{n}^{+}$and forcing with $(\mathbb{R} * T) \times \mathbb{S}_{n}$ does not add sets which are hereditarily of size $\leq \kappa_{n}^{+}$, once again we have $U_{n} \in V$. The generic filters $K_{i}$ are defined as before.

We want to prove that forcing with $\mathbb{R} * \mathbb{P}$ we obtain the desired model. Let be $\mathbb{S}:=\operatorname{Coll}\left(\omega,<\kappa_{0}\right) \times \mathbb{S}_{0}$, and let $G_{\mathbb{S}_{0}}$ be a generic filter for $\mathbb{S}_{0}$ over $V$. As before, we denote by $\mathbb{P}^{*}$ the poset whose conditions are the conditions $p$ of $\mathbb{P}$ such that the $S$-part of $p$ is in $G_{\mathbb{S}_{0}}$.

We will need the following lemma.

LEMma 6.1. $T$ is $\lambda^{+}$-strategically closed in $V^{\mathbb{R}}$.

Proof. $T$ is $\lambda^{+}$-strategically closed if Even has a winning strategy in the game $G_{\lambda^{+}}(T)$ where two players Odd and Even take turns to play conditions $t_{\beta}$ for $\lambda^{+}$many moves with Odd playing at odd stages and Even playing at even and limit stages. Even must play the maximal condition of $T$ at move zero and, at move $\beta$, the condition $t_{\beta}$ must be stronger than any condition played until then. Even wins the game if he can respond at any move. We describe the strategy as follows. At each move $\beta$, Even chooses a condition $r_{\beta} \in \mathbb{R}$ in addition to $t_{\beta} \in T$ in such a way that $t_{\beta} \in r_{\beta}$ and $h t\left(r_{\beta}\right)=\operatorname{dom}\left(t_{\beta}\right)+1$. It follows that for every $\beta$ even or limit, the pair $\left(r_{\beta}, t_{\beta}\right)$ belongs to $\mathbb{Q}$. The closure of $\mathbb{Q}$ ensures that Even can chose $\left(r_{\beta}, t_{\beta}\right)$ at each stage. $\dashv$

THEOREM 6.2. The tree property at $\aleph_{\omega^{2}+1}$ fails in $V^{\mathbb{R} * \mathbb{P}}$.

Proof. $T$ is $\lambda^{+}$-Suslin in $V^{\mathbb{R}}$, we prove that forcing with $\mathbb{S} * \mathbb{P}^{*}$ over $V^{\mathbb{R}}$ does not add a cofinal branch to $T$; in particular $T$ remains Aronszajn in $V^{\mathbb{R} * \mathbb{P}}$ which is a submodel of $V^{\mathbb{R} * \mathbb{S} * \mathbb{P}^{*}}$. The forcing $\mathbb{S}$ is a product of a forcing of size $\kappa_{0}$, namely Coll $\left(\omega,<\kappa_{0}\right)$, with a $\sigma$-closed forcing, namely $\mathbb{S}_{0}$. $\mathbb{S}_{0}$ is $\sigma$-closed, hence by Lemma 2.2 it cannot add cofinal branches to $T$ over $V^{\mathbb{R}}$. The poset $\operatorname{Coll}\left(\omega,<\kappa_{0}\right)$ is $\lambda^{+}$. Knaster in $V^{\mathbb{R} * \mathbb{S}_{0}}$, therefore it cannot add cofinal branches to $T$ over $V^{\mathbb{R} * \mathbb{S}_{0}}$. It follows that $T$ remains Aronszajn in $V^{\mathbb{R} * \mathbb{S}}$.

Claim 6.3. $\mathbb{P}^{*}$ is $\lambda^{+}$-Knaster in $V^{\mathbb{R} * \mathbb{S}}$.

Proof. Given a sequence $\left\langle p_{\beta} ; \beta<\lambda^{+}\right\rangle$of conditions in $\mathbb{P}^{*}$, there exists $n<\omega$ and a stationary set $S^{*} \subseteq \lambda^{+}$such that $p_{\beta}$ has length $n$ for every $\beta \in S^{*}$. The possible stems of $\mathbb{P}^{*}$ length $n$ are $\leq \kappa_{n+1}<\lambda^{+}$hence there exists $s$ and a stationary subset $S \subseteq S^{*}$ such that stem $\left(p_{\beta}\right)=s$ for every $\beta \in S$. Two conditions of $\mathbb{P}^{*}$ with the same stem are compatible, hence the conditions in the subsequence $\left\langle p_{\beta} ; \beta \in S\right\rangle$ are pariwise compatible.

It follows that $\mathbb{P}^{*}$ cannot add cofinal branches to $T$, hence $T$ remains Aronszajn in $V^{\mathbb{R} * \mathbb{S} * \mathbb{P}^{*}}$. 
THEOREM 6.4. $\quad V^{\mathbb{R} * \mathbb{P}} \models \Delta_{\aleph_{\omega^{2}}, \aleph_{\omega^{2}+1}}$.

To prove the theorem we want to use the old argument from Magidor and Shelah's paper, the main difficulty is to deal with the presence of the generic Suslin tree T. For the argument to work, we need $T$ to be $\lambda^{+}$-c.c. in $V^{\mathbb{R} * \mathbb{S}_{n}}$; this motivates the following lemma.

Lemma 6.5. For every $n<\omega$, the tree T remains $\lambda^{+}$-Suslin in $V^{\mathbb{R} * \mathbb{S}_{n}}$.

Proof. We work in $V^{\mathbb{R}}$. Let be $s \in \mathbb{S}_{n}$ and $\dot{A}$ such that

$$
s \Vdash \dot{A} \text { is a maximal antichain in } T \text {. }
$$

We let $\theta$ be some regular cardinal much larger than any cardinal under discussion and let $\mathcal{H}_{\theta}$ be some expansion of $\left\langle H_{\theta}, \in\right\rangle$ by at most countably many constants, functions and relations including $s, T, \mathbb{S}_{n}$ and everything relevant to this proof. We fix a $\lambda^{+}$-approximating sequence, namely a continuous increasing sequence $\left\langle M_{\alpha}\right\rangle_{\alpha \in \lambda^{+}}$of elementary substructures of $\mathcal{H}_{\theta}$ of size $<\lambda^{+}$such that for all $\alpha$, $\lambda^{+} \in M_{\alpha},\left\langle M_{\beta} ; \beta \leq \alpha\right\rangle \in M_{\alpha+1}$ and $M_{\alpha} \cap \lambda^{+}$is an ordinal of $\lambda^{+}$.

By Lemma 6.1 $T$ is $\lambda^{+}$-strategically closed, we assume that every $M_{\alpha}$ contains the corresponding strategy $\tau$.

ClaIm 6.6. Given a model $M$ in the approximating sequence, for every $k \geq n$, $q \in \mathbb{S}_{n}$ and $x \in T \cap M$, there exists $t^{*}>x$ in $T \cap M$ and a condition $q^{*} \leq q$ with $q^{*} \uparrow k=q \uparrow k$ such that $q^{*} \Vdash t^{*}$ is above some element of $\dot{A}$.

Proof. Let $\left\{p_{\gamma} ; \gamma<\kappa_{k}\right\}$ enumerate all the sequences $\bar{q} \uparrow k$ for $\bar{q} \leq q$. We inductively define a decreasing sequence of conditions $\left\langle q_{\alpha}\right\rangle_{\alpha \in \kappa_{k}}$ in $\mathbb{S}_{n} \cap M$ and an increasing sequence of nodes $\left\langle t_{\alpha}\right\rangle_{\alpha \in \kappa_{k}}$ in $T \cap M$ such that

(1) $q_{0}:=q$ and $t_{0}:=x$,

(2) $q_{\alpha} \uparrow k=q \uparrow k$ for every $\alpha$

moreover, we make sure that the nodes are chosen according to the strategy $\tau$. For $\alpha$ limit ordinal, we let $q_{\alpha}$ be the union of all $q_{\beta}$ where $\beta<\alpha$, and $t_{\alpha}$ is the node given by the strategy $\tau$ applied to $\left\langle t_{\beta}\right\rangle_{\beta<\alpha}$. Suppose that $q_{\alpha}$ and $t_{\alpha}$ are defined, we define $q_{\alpha+1}$ and $t_{\alpha+1}$ as follows. We denote by $q_{\alpha} * p_{\alpha}$ the unique condition $r \in \mathbb{S}_{n}$ such that $r \nmid k=p_{\alpha}$ and $r(i)=q_{\alpha}(i)$ for all $i \geq k$. Let $\varphi_{\alpha}$ be the statement:

"There is a condition $\bar{q}$ compatible with $q_{\alpha} * p_{\alpha}$ and there is a node $t>t_{\alpha}$ such that $\bar{q} \Vdash t$ is above some element of $\dot{A}$."

If the statement is true, then we let $t_{\alpha+1}>t$ be the node given by the strategy $\tau$ and we let $q_{\alpha+1}$ be given by

$$
q_{\alpha+1}(i):= \begin{cases}q_{\alpha}(i) & \text { if } i<k, \\ q_{\alpha}(i) \cup \bar{q}(i) & \text { otherwise. }\end{cases}
$$

If the statement is false, we let $q_{\alpha+1}$ be $q_{\alpha}$ and we let $t_{\alpha+1}$ be $t_{\alpha}$.

Using the closure of $\mathbb{S}_{k}$, let $q^{*}$ be a lower bound for $\left\langle q_{\alpha} ; \alpha \in \kappa_{k}\right\rangle$ (such a lower bound exists because the conditions in the sequence have the same $k$-lower part). We also let $t^{*}$ be the node given by the strategy $\tau$ applied to $\left\langle t_{\alpha} ; \alpha \in \kappa_{k}\right\rangle$. By elementarity of $M$ we can assume that both $q^{*}$ and $t^{*}$ belong to $M$. We show that

$$
q^{*} \Vdash t^{*} \text { is above some element of } \dot{A} \text {. }
$$


Suppose otherwise, we will reach a contradiction. $q^{*}$ forces that $\dot{A}$ is a maximal antichain, so we have

$$
q^{*} \Vdash t^{*} \text { is compatible with some element of } \dot{A} \text {. }
$$

Let be $t^{* *}$ and $q^{* *}$ be such that $t^{* *}$ is compatible with $t^{*}, q^{* *} \leq q^{*}$ and $q^{* *}$ forces that $t^{* *}$ is in $\dot{A}$. The sequence $q^{* *} \uparrow k$ appears in the enumeration as $p_{\alpha}$ for some $\alpha$, so by construction $q^{* *}$ is compatible with $q_{\alpha} * p_{\alpha}$ and $t^{* *}$ is compatible with $t_{\alpha}$. It follows that the statement $\varphi_{\alpha}$ is true, hence $t_{\alpha+1}$ and $q_{\alpha+1}$ were defined so that $t_{\alpha+1}>t_{\alpha}$ and

$$
q_{\alpha+1} \Vdash t_{\alpha+1} \text { is above some element of } \dot{A} \text {. }
$$

This prove that $q^{* *}$ and $t^{* *}$ are as required.

We resume the proof of the lemma. Using the claim, we can inductively define a decreasing sequence $\left\langle s_{\alpha}\right\rangle_{\alpha<\lambda^{+}}$such that

(a) $s_{0}:=s$ and $s_{\alpha} \uparrow k=s \uparrow k$ for every $\alpha$,

(b) for every $x \in T \cap M_{\alpha}$ there exists $y>x$ in $T \cap M_{\alpha}$ such that

$$
s_{\alpha} \Vdash y \text { is above some element of } \dot{A} \text {. }
$$

Finally let be $s_{\infty}:=\bigcup_{\alpha<\lambda^{+}} s_{\alpha}$ and $M_{\infty}:=\bigcup_{\alpha<\lambda^{+}} M_{\alpha}$. We show that

$$
s_{\infty} \Vdash \text { every element of } \dot{A} \text { can be extended to an element of } T \cap M_{\infty} \text {; }
$$

this will complete the proof as $T \cap M_{\infty}$ belongs to $V^{\mathbb{R}}$ where $T$ is $\lambda^{+}$-Suslin. Assume $s_{\infty} \Vdash \dot{x} \in \dot{A}$, we take $s \leq s_{\infty}$ and $x \in T$ such that $s \Vdash \dot{x}=x$. By elementarity we can assume without loss of generality that $x \in M_{\infty}$. Let $\alpha$ be the least ordinal such that $x \in M_{\alpha}$. By $(b)$ there exists $y>x$ in $T \cap M_{\alpha}$ such that

$$
s_{\alpha} \Vdash y \text { is above some element of } \dot{A} \text {. }
$$

In particular, $s_{\infty}$ forces the same. By maximality of $\dot{A}$ we have $s_{\infty} \Vdash y$ is above $\dot{x}$ that completes the proof of the lemma.

We are now ready to prove Theorem 6.4. From now on, we will essentially follow the arguments of [5] with minor adjustments.

Let $G_{\mathbb{R}}$ be an $\mathbb{R}$-generic filter over $V$, let $G_{T}$ be a $T$-generic filter over $V\left[G_{\mathbb{R}}\right]$ and let $G_{n}$ be an $\mathbb{S}_{n}$-generic filter over $V$. In $V\left[G_{\mathbb{R}}\right]$ we let $p \in \mathbb{P}, \dot{A}, \dot{S}$ and $\mu<\lambda$ be such that the $S$-part of $p$ is in $G_{n}$ and

$p \Vdash \dot{A}$ is an algebra on $\lambda^{+}$with $\mu$ operations and $\dot{S} \subseteq \lambda^{+}$is a stationary set.

Let $l<\omega$ be such that $\mu<\kappa_{l}$, we can assume without loss of generality that the length of $p$ is $n>l$ and $p$ forces that every ordinal in $\dot{S}$ has cofinality $<\kappa_{l}$. In any $\mathbb{P}$-generic extension $V\left[G_{\mathbb{R}}\right]\left[G_{\mathbb{P}}\right]$ we can observe that for every $\beta$ in $\dot{S}$ there is a condition $q_{\beta} \in G_{\mathbb{P}}$ such that $q_{\beta} \Vdash \beta \in \dot{S}$. Since there are less than $\lambda^{+}$many possible stems of $q_{\beta}$, there is a stationary subset $\dot{E}$ of $\dot{S}$ such that for every $\beta \in \dot{E}$ the stem of $q_{\beta}$ is fixed. We can assume without loss of generality that the stem of $p$ extends this fixed stem, hence $p$ forces that

$$
\dot{E}=\left\{\beta<\lambda^{+} ; \exists q \leq p \text { in } G_{\mathbb{P}} \text { such that } \operatorname{stem}(q)=\operatorname{stem}(p) \text { and } q \Vdash \beta \in \dot{S}\right\}
$$
is stationary in $\lambda^{+}$.

In the rest of the proof we will work in $V\left[G_{\mathbb{R}} * G_{T}\right]\left[G_{n}\right]$, recall that $\kappa_{n}$ is still supercompact in this model. We define $\mathbb{P}_{n}^{*}$ to be the set of all conditions $q \in \mathbb{P}$ of 
length $\geq n$ such that the $S_{n}$-part of $q$ belongs to $G_{n}$. We let

$E^{*}:=\left\{\alpha<\lambda^{+} ; \exists q \leq p\right.$ in $\mathbb{P}_{n}^{*}$ with the same stem of $p$ such that $\left.q \Vdash \alpha \in \dot{E}\right\}$.

LEMma 6.7. $V\left[G_{\mathbb{R}}\right]\left[G_{T}\right]\left[G_{n}\right] \models E^{*}$ is a stationary subset of $\lambda^{+}$.

Proof. Note that $V\left[G_{\mathbb{R}}\right]\left[G_{T}\right]\left[G_{n}\right]=V\left[G_{\mathbb{R}}\right]\left[G_{n}\right]\left[G_{T}\right]$. Assume for a contradiction that we can find in $V\left[G_{\mathbb{R}}\right]\left[G_{n}\right]\left[G_{T}\right]$ a club $C \subseteq \lambda^{+}$which is disjoint from $E^{*}$. By Lemma $6.5, T$ is $\lambda^{+}$-c.c., so there exists a club $D \subseteq C$ that lies in $V\left[G_{\mathbb{R}}\right]\left[G_{n}\right]$, thus we can assume without loss of generality that $C$ is in $V\left[G_{\mathbb{R}}\right]\left[G_{n}\right]$. We work in $V\left[G_{\mathbb{R}}\right]$. As we did for $\mathbb{S}_{0}$ in the previous section, we define an equivalence relation $\sim$ on $\mathbb{S}_{n}$ by

$$
\left\langle g_{i}\right\rangle_{n-1<i} \sim\left\langle h_{i}\right\rangle_{n-1<i} \Longleftrightarrow \text { for large enough } i, g_{i}=h_{i} .
$$

For a condition $r \in \mathbb{S}_{n}$, we denote by $[r]$ its equivalence class. Let $H_{n}:=\{[r] ; r \in$ $\left.G_{n}\right\}$, then we have

$$
V\left[G_{\mathbb{R}}\right] \subseteq V\left[G_{\mathbb{R}}\right]\left[H_{n}\right] \subseteq V\left[G_{\mathbb{R}}\right]\left[G_{n}\right] .
$$

The same arguments for the proof of Proposition 4.5 show that $\mathbb{S}_{n} / \sim$ is $\lambda^{+}$-c.c.

Therefore, we can assume that $C$ lies in $V\left[G_{\mathbb{R}}\right]\left[H_{n}\right]$. We fix a generic $G_{\mathbb{P}}$ for $\mathbb{P}$ over $V\left[G_{\mathbb{R}}\right]$ and we let $E$ be the interpretation of $\dot{E}$ in this model. We observe that $V\left[G_{\mathbb{R}}\right]\left[G_{\mathbb{P}}\right]$ is obtained by forcing with $\mathbb{P}_{n}^{*}$ over $V\left[G_{\mathbb{R}}\right]\left[G_{T}\right]\left[G_{n}\right]$. By the definition of $E^{*}$ we have $E \subseteq E^{*}$. By Lemma 4.4 we have $V\left[G_{\mathbb{R}}\right]\left[H_{n}\right] \subseteq V\left[G_{\mathbb{R}}\right]\left[G_{\mathbb{P}}\right]$, in particular $C$ belongs to $V\left[G_{\mathbb{R}}\right]\left[G_{\mathbb{P}}\right]$, but $C$ is disjoint from $E^{*}$, hence from $E$. That contradicts the fact that $E$ is stationary in $V\left[G_{\mathbb{R}}\right]\left[G_{\mathbb{P}}\right]$.

Now we define in $V\left[G_{\mathbb{R}}\right]\left[G_{T}\right]\left[G_{n}\right]$ a forcing notion $\mathbb{C}_{n}$ whose conditions are sequences $\left\langle\alpha_{0}, g_{0}, f_{0}, \ldots, \alpha_{n-1}, g_{n-1}, f_{n-1}, s\right\rangle$ such that

(1) $\left\langle\alpha_{0}, \ldots, \alpha_{n-1}\right\rangle$ is the $\alpha$-part of $p$;

(2) $\left\langle f_{0}, \ldots, f_{n-1}\right\rangle$ belongs to $\prod_{i<n} \operatorname{Coll}\left(\alpha_{i}^{+\omega+2}, \kappa_{i}\right)$;

(3) $\left\langle g_{0}, \ldots, g_{n-1}\right\rangle$ belongs to $\prod_{i<n} \operatorname{Coll}\left(\kappa_{i-1}^{+\omega+2},<\alpha_{i}\right)$;

(4) $s \in \operatorname{Coll}\left(\kappa_{n}^{++},<\kappa_{n}\right)$.

The ordering is:

$$
\left\langle\alpha_{0}, g_{0}, f_{0}, \ldots, \alpha_{n-1}, g_{n-1}, f_{n-1}, s\right\rangle \leq\left\langle\alpha_{0}, g_{0}^{\prime}, f_{0}^{\prime}, \ldots, \alpha_{n-1}, g_{n-1}^{\prime}, f_{n-1}^{\prime}, s^{\prime}\right\rangle
$$

if and only if for all $i, f_{i} \leq f_{i}^{\prime}, g_{i} \leq g_{i}^{\prime}$ and $s \leq s^{\prime}$. Let $G_{\mathbb{C}_{n}}$ be any generic filter for $\mathbb{C}_{n}$ that contains the stem of $p$. In $V\left[G_{\mathbb{R}}\right]\left[G_{T}\right]\left[G_{n}\right]\left[G_{\left.\mathbb{C}_{n}\right]}\right.$ we define $\mathbb{P}_{n}^{* *} \subseteq \mathbb{P}_{n}^{*}$ as the set of all conditions $q$ of length $n$ such that the stem of $q$ is in the generic $G_{\mathbb{C}_{n}}$ for $\mathbb{C}_{n}$. Every two elements of $\mathbb{P}_{n}^{* *}$ are compatible. Moreover, by the closure of $\mathbb{S}_{n} \times \mathbb{C}_{n}$ we have that $\mathbb{P}_{n}^{* *}$ is $\sigma$-closed. Both $\mathbb{P}_{n}^{*}$ and $\mathbb{P}_{n}^{* *}$ satisfy the property that for every statement $\varphi$ in the forcing language of $\mathbb{P}$ there exists $r \in \mathbb{P}_{n}^{*}$, respectively $r \in \mathbb{P}_{n}^{* *}$, such that $r \leq p$ and $r$ decides $\varphi$ (see [5, Lemmas 6 and 8]).

We are going to define an algebra $A^{*}$ in $V\left[G_{\mathbb{R}}\right]\left[G_{T}\right]\left[G_{n}\right]\left[G_{\mathbb{C}_{n}}\right]$ that will represent a version of the algebra $\dot{A}$. Without loss of generality we can assume that the order type of $\dot{A}$, namely the sequence of the cardinals specifying for each $n$ how many $n$-ary operations are in $\dot{A}$, is in $V[\mathbb{R}]$. The algebra $A^{*}$ will be generated by finite sequences of ordinals less than $\lambda^{+}$. We must specify for each two terms, whether they denote the same element of the algebra, thus the elements of $A^{*}$ are actually the equivalence classes of terms. Suppose that $\dot{A}$ can be written as $\left\langle\lambda^{+},\left\langle\dot{o}_{i}\right\rangle_{i<\mu}\right\rangle$. We can assume without loss of generality that one of the operations of $\dot{A}$ is the 
identity on the terms of the algebra. Given $\rho, \eta<\mu$ and given two sequences of ordinals $\vec{\beta}$ and $\vec{\gamma}$ in $\lambda^{+}$, we let $\rho(\vec{\beta})={ }^{*} \eta(\vec{\gamma})$ if some condition $q$ in $\mathbb{P}_{n}^{* *}$ forces that $\dot{o}_{\rho}(\vec{\beta})=\dot{o}_{\eta}(\vec{\gamma})$ (in the sense of the forcing language for $\mathbb{P}$ ). The elements of $A^{*}$ are the equivalence classes under the relation $={ }^{*}$. Note that $A^{*}$ is well defined because any two conditions of $\mathbb{P}_{n}^{* *}$ are compatible.

We define an ordering on $A^{*}$ by letting $\rho(\vec{\beta})<^{*} \eta(\vec{\gamma})$ if some condition of $\mathbb{P}_{n}^{* *}$ forces that $\dot{o}_{\rho}(\vec{\beta})<\dot{o}_{\eta}(\vec{\gamma})$ as ordinals.

Lemma 6.8 ([5, Lemmas 9, 10, 11]). $A^{*}$ is well ordered by $<^{*}$ in order type $\lambda^{+}$.

Now we work in $V\left[G_{\mathbb{R}}\right]\left[G_{T}\right]\left[G_{n}\right]$ where we let $\dot{A}^{*}$ be a $\mathbb{C}_{n}$-term for the algebra $A^{*}$. Let $U^{*}$ be the interpretation of $\dot{F}_{n}$ in this model (recall that $\dot{F}_{n}$ was an $(\mathbb{R} * T) \times \mathbb{S}_{n}$ name for a normal ultrafilter on $\mathcal{P}_{\kappa_{n}}\left(\lambda^{+}\right)$and $U_{n}$ is the projection of such an ultrafilter). We let $j$ be the supercompact elementary embedding corresponding to $U^{*}$. We consider $\theta$ a regular cardinal much larger than any cardinal under discussion and we let $\mathcal{H}_{\theta}:=\left\langle H_{\theta}, \lambda^{+}, E^{*}, \mathbb{P}_{n}^{*}, p, \mathbb{C}_{n}, \dot{A}^{*}\right\rangle$. We define

$$
B:=\left\{M \cap \lambda^{+} ; M \prec \mathcal{H}_{\theta},|M|<\kappa_{n}, M \cap \lambda^{+} \in \bigcap_{W \in U^{*} \cap M} W\right\} .
$$

Since $j\left[\lambda^{+}\right] \in j(B)$, we have $B \in U^{*}$. We let

$$
\begin{aligned}
B^{*}:= & \left\{X \in \mathcal{P}_{\kappa_{n}}\left(\lambda^{+}\right) ; X \cap \kappa_{n} \text { is inaccessible, o.t. }(X)=\left(X \cap \kappa_{n}\right)^{+\omega+1},\right. \\
& \left.E^{*} \cap X \text { is stationary in } \sup (X)\right\},
\end{aligned}
$$

then $B^{*}$ also belongs to $U^{*}$.

Lemma 6.9. Let $X \in B \cap B^{*}$ such that $X \cap \kappa_{n} \in A_{n}^{p}$ and let $M \prec \mathcal{H}_{\theta}$ be witnessing the fact that $X \in B$. There exists a condition $q \in \mathbb{P}_{n}^{*}$ of length $n+1$ such that $\alpha_{n}^{q}=X \cap \kappa_{n}$ and $q$ extends every extension of $p$ in $\mathbb{P}_{n}^{*} \cap M$ with the same stem as $p$.

Proof. The proof is just as in [5, Lemma 13] we include it for the sake of completeness. Let $\alpha_{n}:=X \cap \kappa_{n}$. We use the closure of $\mathbb{S}_{n}$ to define $q$ as

$$
\left\langle\alpha_{0}, g_{0}, f_{0}, \ldots, \alpha_{n-1}, g_{n-1}, f_{n-1}, \alpha_{n}, g_{n}, f_{n}^{*}, A_{n+1}^{*}, g_{n+1}^{*}, F_{n+1}^{*}, \ldots\right\rangle
$$

where

(1) $\alpha_{0}, g_{0}, f_{0}, \ldots, \alpha_{n-1}, g_{n-1}, f_{n-1}, \alpha_{n}, g_{n}$ are like in $p$;

(2) for $j>n, A_{j}^{*}:=\bigcap_{W \in U_{j} \cap M} W$;

(3) for $j>n, g_{j}^{*}$ is the union of the $j$-th components of $G_{n} \cap M$;

(4) $f_{n}^{*}:=\bigcup\left\{F\left(\alpha_{n}\right) ; F \in M,[F]_{U_{n}} \in K_{n}\right\}$;

(5) for $j>n, F_{j}^{*}(\beta):=\bigcup\left\{F(\beta) ; F \in M,[F]_{U_{j}} \in K_{j}\right\}$.

Every $A_{j}^{*}$ belongs to $U_{j}$ because the cardinality of $M$ is less than $\kappa_{n}<\kappa_{j}$ (moreover, $U^{*} \cap M$ belongs to $V$ by the closure of $(\mathbb{R} * T) \times \mathbb{S}_{n}$, so we are intersecting a family of sets in $V$ ).

We show that $f_{n}^{*}$ is in $\operatorname{Coll}\left(\alpha_{n}^{+\omega+2},<\kappa_{n}\right)$ : we assumed that $X$ belongs to every $W \in U_{n}^{*} \cap M$, hence, since $U_{n}$ is the projection of $U_{n}^{*}$, we have $X \cap \kappa_{n}=\alpha_{n} \in W$ for $W \in U_{n} \cap M$. If $F, F^{\prime} \in M$ and $[F]_{U_{n}},\left[F^{\prime}\right]_{U_{n}} \in K_{n}$, then the set $P:=\{\beta<$ $\kappa_{n} ; F(\beta)$ and $F^{*}(\beta)$ are compatible $\}$ belongs to $U_{n}$ and we have $P \in U_{n} \cap M$, so $\alpha_{n} \in P$. It follows that $f_{n}^{*}$ is the union of $|M|$ many mutually compatible conditions of $\operatorname{Coll}\left(\alpha_{n}^{+\omega+2},<\kappa_{n}\right)$ and by the closure of $\mathbb{S}_{n}$ this union is in $V\left[G_{\mathbb{R}}\right]$. We have 
$|M|=|X|=\alpha_{n}^{+\omega+1}$, hence $f_{n}^{*}$ is in $\operatorname{Coll}\left(\alpha_{n}^{+\omega+2},<\kappa_{n}\right)$. The same argument show that the $F_{j}^{*}$ are well defined.

We resume the proof of the theorem. Take $X \in B \cap B^{*}$ and let $M$ witness the fact that $X$ is in $B$. Let $\alpha=X \cap \kappa_{n}=M \cap \kappa_{n}$ and let $q$ be as in Lemma 6.9.

Claim 6.10. $q$ forces that the subalgebra of $\dot{A}$ generated by $X$ has the same order type as $X$ and $X$ is cofinal in it.

Proof. We sketch the proof, for more details see [5, p. 804]. Let $\mathcal{B}^{*}$ be the subalgebra of $\mathcal{A}^{*}$ generated by $X$. It is not difficult to see that $\mathcal{B}^{*}$ has order type $|X|$ and $X$ is cofinal in it. $\mathcal{B}^{*}$ is defined in $V\left[G_{\mathbb{R}}\right]\left[G_{T}\right]\left[G_{n}\right]\left[G_{\mathbb{C}_{n}}\right]$, nevertheless by the $\kappa_{n}$-c.c. of $\mathbb{C}_{n}$ we can see that $\mathcal{B}^{*}$ exists in the smaller model $V\left[G_{\mathbb{R}}\right]\left[G_{T}\right]\left[G_{n}\right][J]$, where $J$ is the generic object added by $G_{n}$ for the set of all conditions in $\mathbb{C}_{n}$ that have as last coordinate a condition in $\operatorname{Coll}\left(\kappa_{n-1},<\alpha_{n}^{q}\right)\left(\right.$ instead of $\left.\operatorname{Coll}\left(\kappa_{n-1},<\kappa_{n}\right)\right)$. By the closure of $(\mathbb{R} * T) \times \mathbb{S}_{n}$, we have $\mathcal{B}^{*} \in V\left[G_{\mathbb{R}}\right][J]$. Now, let $G_{\mathbb{P}} \subseteq \mathbb{P}$ be a generic filter for $\mathbb{P}$ containing the condition $q$. Since the stem of $q$ is in $J$, we have $V\left[G_{\mathbb{R}}\right][J] \subseteq V\left[G_{\mathbb{R}}\right]\left[G_{\mathbb{P}}\right]$. Thus $\mathcal{B}^{*}$ is in $V\left[G_{\mathbb{R}}\right]\left[G_{\mathbb{P}}\right]$. Let $\mathcal{A}:=\left(\lambda^{+},\left\langle o_{\rho}\right\rangle_{\rho<\mu}\right)$ be the interpretation of $\dot{\mathcal{A}}$ by $G_{\mathbb{P}}$ and let $\mathcal{B}$ be the subalgebra of $\mathcal{A}$ generated by $X$. To prove the claim, it suffices to show that $\mathcal{B}$ is isomorphic to $\mathcal{B}^{*}$ via an isomorphism that is the identity on $X$. The isomorphism associates to $o_{\rho}(\vec{\beta})$ the equivalence class of $\rho(\vec{\beta})$, denoted $[\rho(\vec{\beta})]$. To show that this is an isomorphism, consider $[\rho(\vec{\beta})]$ and $[\eta(\vec{\gamma})]$ two terms of $\mathcal{B}^{*}$ and suppose that $[\rho(\vec{\beta})]=[\eta(\vec{\gamma})](\operatorname{resp} .[\rho(\vec{\beta})]<[\eta(\vec{\gamma})])$ in the sense of $\mathcal{B}^{*}$. This means that there exists $r \leq p$ with the same stem as $p$ and there exists $t \in J$ such that, if $r^{*}$ is a condition like $r$ except that the stem is $t$, then $r^{*}$ forces that $\dot{o}_{\rho}(\vec{\beta})=\dot{o}_{\eta}(\vec{\gamma})$ (resp. $\dot{o}_{\rho}(\vec{\beta})<\dot{o}_{\eta}(\vec{\gamma})$ ). By elementarity of $M$, we can assume that $r \in M$, hence $q \leq r$, so $r \in G_{\mathbb{P}}$. By definition of $J$, we have $r^{*} \in G_{\mathbb{P}}$, therefore $o_{\rho}(\vec{\beta})=o_{\eta}(\vec{\gamma})$ (resp. $o_{\rho}(\vec{\beta})<o_{\eta}(\vec{\gamma})$ ). That completes the proof of the claim.

In conclusion, $q$ forces that the order type of $X$ is a regular cardinal. Since $\alpha_{n}^{q}=X \cap \kappa_{n}$, the order type of $X$ is $\left(X \cap \kappa_{n}\right)^{+\omega+1}=\left(\alpha_{n}^{q}\right)^{+\omega+1}$ and no cardinal are collapsed between $\alpha_{n}$ and $\alpha_{n}^{+\omega+2}$. We also note that for $\beta \in E^{*} \cap X$, some extension of $p$ in $\mathbb{P}_{n}^{*}$ with the same stem forces $\beta \in E$, but by elementarity such an extension is in $M$, so $q$ extends it, hence $q$ forces $\beta \in E$. Since $X \in B^{*}$, we have $X \cap E^{*}$ is stationary in the $\sup (X)$, so we get that $q$ forces that the subalgebra generated by $X$ is a witness to $\Delta_{\aleph_{\omega^{2}}, \aleph_{\omega^{2}+1}}$. The other direction is analogous, so that completes the proof of Theorem 6.4 and consequently of Theorem 1.3.

$\S 7$. Acknowledgements. The authors wish to thank the referee for carefully reading our manuscript and for many useful comments. The research of the first author was supported by the European Commission under a Marie Curie Intra-European Fellowship through the project \# 624381 (acronym LAPSCA).

\section{REFERENCES}

[1] P. EkLof, On the existence of $\kappa$ free Abelian groups. Proceedings of the American Mathematical Society, vol. 47 (1975), pp. 65-72.

[2] K. Kunen, Saturated ideals, this Journal, vol. 43 (1978), no. 1, pp. 65-76.

[3] R. LAVER, Making the supercompactness of $\kappa$ indestructible under $\kappa$-directed closed forcing. Israel Journal of Mathematics, vol. 29 (1978), pp. 385-388. 
[4] M. Magidor and S. Shelah, The tree property at successors of singular cardinals. Archive for Mathematical Logic, vol. 35 (1996), no. 5-6, pp. 385-404.

[5] - When does almost free imply free? (For groups, transversals, etc.). Journal of the American Mathematical Society, vol. 7 (1994), no. 4, pp. 769-830.

[6] W. J. Mitchell, Aronszajn trees and the independence of the transfer property. Annals of Mathematical Logic, vol. 5 (1972), pp. 21-46.

[7] I. NeEman, The tree property up to $\aleph_{\omega+1}$, this JournaL, vol. 79 (2014), pp. 429-459.

[8] S. SHELAh, A compactness theorem for singular cardinals, free algebras, Whitehead problem and transversals. Israel Journal of Mathematics, vol. 21 (1975), pp. 319-339.

[9] - On successors of singular cardinals. Logic Colloquium (M. Boffa, D. Van Dallen, and K. McAloon, editors), Studies in Logic and the Foundations of Mathematics, vol. 78, North Holland, Amsterdam, 1979, pp. 357-380.

[10] D. Sinapova, The tree property at $\aleph_{\omega+1}$, this JournaL, vol. 77 (2012), no. 1, pp. 279-290.

[11] S. Unger, Fragility and indestructibility of the tree property. Archive for Mathematical Logic, vol. 51 (2012), no. 5-6, pp. 635-645.

\section{HEBREW UNIVERSITY OF JERUSALEM \\ EINSTEIN INSTITUTE OF MATHEMATICS \\ EDMOND J. SAFRA CAMPUS \\ GIVAT RAM, 91904 JERUSALEM, ISRAEL}

E-mail: laura.fontanella@mail.huji.ac.il

E-mail:mensara@savion.huji.ac.il 